\title{
Lista anotada de los Euchromiina "polillas avispas" (Lepidoptera: Erebidae: Arctiinae: Arctiini) del departamento de Loreto (Perú), con el reporte de nue- vos registros y sinónimos
}

\section{Annotated list of the Euchromiina "wasp moths" (Lepidoptera: Erebidae: Arctiinae: Arctii- ni) from the department of Loreto (Peru), with the report of new records and synonyms}

Juan Grados* 1

https://orcid.org/0000-0002-2277-3616

gradosjuan@hotmail.com

Juan José Ramírez ${ }^{2}$

https://orcid.org/0000-0001-5760-5172

macrodontia@hotmail.com

\section{* Corresponding author}

1 Universidad Nacional Mayor de San Marcos, Museo de Historia Natural, Departamento de Entomología, Lima, Perú.

2 Universidad Nacional de la Amazonía Peruana, Calle Sargento Lores, 385, Iquitos, Perú.

\section{Citación}

Grados J, Ramírez JJ. 2021. Lista anotada de los Euchromiina "polillas avispas" (Lepidoptera: Erebidae: Arctiinae: Arctiini) del departamento de Loreto (Perú), con el reporte de nuevos registros y sinónimos. Revista peruana de biología 28(especial): $\mathbf{e 2 1 9 0 6} 001$ - 018 (Diciembre 2021). doi: http://dx.doi. org/10.15381/rpb.v28iespecial.21906

$\begin{array}{ll}\text { Presentado: } & 09 / 02 / 2021 \\ \text { Aceptado: } & 10 / 08 / 2021 \\ \text { Publicado online: } & 30 / 12 / 2021\end{array}$

Editor:

Leonardo Romero

\section{Resumen}

Para el departamento de Loreto se registra 113 especies de Euchromiina (Lepidoptera: Arctiinae). Además, se reportan 9 nuevos registros para Perú: Sphecosoma abnormis, Isanthrene profusa, Autochloris collocata, A. consociata, Andrenimorpha lycopolis, Leucotmemis torrida, Cosmosoma contracta, Pseudomya biradiata y $P$. cretheis. Se proponen dos nuevos sinónimos: Diptilon aurantiipes Rothschild como sinónimo más reciente de Sphecosoma abnormis Hampson; Isanthrene flavizonata Gaede como sinónimo más reciente de Tigrinadia quadricincta Kaye. Esta última especie por ahora es conocida solo de Perú. Se describe un nuevo tipo de órgano androconial en la parte ventral del abdomen.

\section{Abstract}

For the department of Loreto, 113 species of Euchromiina (Lepidoptera: Arctiinae) are recorded. In addition, 9 new records are reported for Peru: Sphecosoma $a b$ normis, Isanthrene profusa, Autochloris collocata, A. consociata, Andrenimorpha lycopolis, Leucotmemis torrida, Cosmosoma contracta, Pseudomya biradiata and $P$. cretheis. Two new synonyms are proposed: Diptilon aurantiipes Rothschild as a junior synonym for Sphecosoma abnormis Hampson; Isanthrene flavizonata Gaede as a junior synonym for Tigrinadia quadricincta Kaye. This last species for now is known only from Peru. A new type of androconial organ is described in the ventral part of the abdomen.

Palabras clave:

Amazonia; nuevo registro; nuevo sinónimo; órgano androconial; taxonomía.

Keywords:

Amazonia; new record; new synonym; androconial organ; taxonomy.

\section{Introducción}

La historia taxonómica de la subtribu Euchromiina (Artiini) está ligada con los Ctenuchina. Conocidas comúnmente como "polillas avispas", con el transcurrir del tiempo han sido consideradas en varios niveles taxonómicos, basados en morfología externa y venación alar. Tratados por Kirby (1837) como familia Ctenuchidae, en tanto Hampson (1898) los trató como Syntomidae y años después como Amatidae (Hampson, 1914). Fueron tratados por Forbes (1939) como Euchromiidae con tres subfamilias: Amatinae, Ctenuchinae y Euchromiinae; en tanto Flemming (1959) consideró a los Ctenuchina y Euchromiina como subfamilias dentro de los Ctenuchidae. 
A fines del siglo pasado, Kitching y Rawlins (1998) propusieron que ambos grupos pertenecían a los Arctiidae, tratándolos como tribus, estatus seguido por Jacobson y Weller (2002). En base a evidencias moleculares, se propuso que los Arctiinae pertenecerían a la familia Erebidae (Zahiri et al. 2010), tratados igualmente por Lafontaine \& Schmidt (2010). En un trabajo molecular sobre la filogenia de los Erebidae (Zahiri et al. 2012), corroboraron su propuesta, considerando a los Arctiinae como una subfamilia de los Erebidae (Noctuoidea) con tres linajes: Lithosiini, Syntomini y Arctiini. Esta última con siete subtribus: Arctiina, Spilosomina, Callimorphina, Pericopina, Phaegopterina, Ctenuchina y Euchromiina (Lafontaine \& Fibiger 2006, Zahiri et al. 2010, Lafontaine \& Schmidt 2010).

Los Euchromiina es un grupo de Arctiinae con distribución fundamentalmente neotropical. Comprenden 758 especies en 58 géneros (Zerny 1912), sin incluir el género Euchromia que ocurre en la parte afrotropical y región indoautraliana (Common 1970, Vives 2019). Especies de algunos géneros como Myrmecopsis Newman, Sphecosoma Butler presentan similaridad morfológica con especies de avispas (Klages 1906, Kaye, 1913) lo que podría estar relacionado con complejos miméticos. Lo mismo sucede con especies de Pseudopompilia Druce (=Phaeosphecia Hampson), Pleurosoma Orfila y Pompiliodes Hampson, considerando además que son de hábitos diurnos y en algunos casos el comportamiento de vuelo es muy parecido.

Los machos de un buen número de especies de Arctiinae poseen órganos androconiales en diferentes partes del cuerpo: las bolsas subabdominales, modificaciones de los segmentos abdominales II y III (Barth 1953, Weller et al. 2000), encontrados en los géneros Myrmecopsis, algunas especies de Cosmosoma Hübner y Sarosa Walker entre otros; modificaciones de los segmentos II, III y IV de la parte ventral del abdomen, típico del género Loxophlebia Butler y Mesothen Druce; presencia de órganos androconiales en las patas de Orcynia calcarata y en algunas especies de Pheia Walker y Chrysocale Walker; por último, presencia de coremata en algunas especies de los géneros Pleurosoma Orfila y Cosmosoma Hübner (Grados datos inéditos).

Por ahora, no existe un trabajo filogenético del grupo y solo se cuenta con la revisión de algunos géneros: Horama Hübner (Dietz \& Duckworth 1976), Macrocneme Hübner (Dietz 1994), Psoloptera Butler (Simmons 2006) Mallodeta y Erruca (Pinheiro \& Duarte 2010). En las últimas dos décadas, algunas especies nuevas han sido descritas (Simmons 2004, Cerda 2008, Grados, 2015) y existen algunos trabajos faunísticos llevados a cabo en México, Perú y Brasil (Hernádez-Baz \& Grados 2004, Grados 1999, 2001, Teston \& Gianluppi 2019).

El departamento de Loreto es el más grande del Perú y forma parte de la Amazonía peruana, considerada como una región con alta riqueza de especies. En general, en el departamento se han realizado inventarios focalizados en plantas y vertebrados (Vriesendorp et al. 2006, Vriesendorp et al. 2007, Pitman et al. 2011), mientras que, son escasos los trabajos sobre lepidópteros, pudiendo señalar uno sobre mariposas y otro sobre polillas (Lamas et al. 1996, Racheli \& Callegari 1996).

Presentamos la lista de especies de los Euchromiina del departamento de Loreto, con anotaciones de algunas especies. Hemos añadido algunas especies que tienen como localidad tipo algún lugar de Loreto, basados en las descripciones originales e información de los tipos. Por último, presentamos fotografías de los adultos machos, en vista dorsal y ventral, de algunos nuevos registros, así como la morfología de los genitales, en caso no estén publicadas con anterioridad. Esto permitirá definir mejor las diferencias con especies similares.

\section{Material y métodos}

Las muestras fueron recolectadas con trampas de luz, utilizando en algunos casos lámparas de vapor de mercurio, lámparas de luz mixta, plantas de Heliotropium sp. (Borraginaceae) y redes entomológicas para las especies de hábitos diurnos. Las recolectas han sido llevadas a cabo en diferentes lugares, comprendiendo además los extremos de los cuatro puntos cardinales del departamento: Yurimaguas hacia el occidente, Cochiquinas en el oriente, Tres Fronteras (río Putumayo) en la parte septentrional y, Quebrada Tunuya en la austral, casi frontera con Pucallpa.

Las colecciones revisadas fueron:

MHNP: Muséum National d'Histoire Naturelle, Paris, Francia.

MUSM: Museo de Historia Natural, Universidad Nacional Mayor de San Marcos, Lima, Perú.

NHMUK: Natural History Museum United Kingdom, LLondres, Reino Unido.

ZMHB: Zoologisches Museum, Humboldt Universität, Berlin, Alemania.

UMO: University Museum, Oxford University, Oxford, Reino Unido.

Los genitales fueron diseccionados y preparados utilizando una solución de KOH (10\%) en baño maría. Para la mejor observación de los caracteres, se utilizó como solución de tinción negro de chlorazol (Cannon 1937, 1941, Carayon 1969). Las fotografías de los adultos se tomaron con una cámara Nikon D80 y los genitales con una cámara Canon EOS Rebel T6 y un macro Canon MP-E 65mm.

\section{Resultados}

La secuencia de los géneros está de acuerdo con el orden filético propuesto por Hampson (1898) y Draudt (1916-1919). Las especies dentro de los géneros están en orden alfabético. Para cada especie, se presenta el autor y el año de publicación. En otra columna, con números ordinales, se presentan las localidades donde se han registrado las especies (Tabla 1). Para cada localidad se proporciona la altura y coordenadas geográficas (latitud y longitud). En algunos casos, para lugares muy cercanos, se fusionaron en un solo punto. 
Las especies con ciertas anotaciones están consignadas con superíndices numerales. Las especies que han sido incluidas en base a las descripciones originales están señaladas con cruz como superíndice. Los registros del género Macrocneme Hübner fueron tomados de Dietz (1994).

Se registran 113 especies que corresponden a 39 géneros: 32 especies con solo un lugar de recolecta. Destacan Pseudomya biradiata, al parecer una especie no muy común; Mimagyrta abdominalis y Agyrtiola niepelti, ambas de hábitos diurnos y que al parecer no llegan a las trampas de luz. Por otro lado, se tuvo las especies con amplia distribución, como Autochloris completa, Sarosa acutior, Pseudomya temenis e Hypocharis clusia. Las tres primeras con amplia distribución en toda la Amazonía peruana y, la última, especie común y registrada solo en Loreto (Tabla 1).

Se tuvo 105 localidades con registros, sin contar con las localidades que no se tiene coordenadas geográficas y que provienen de las descripciones originales (Tabla. 2, Fig. 1).

Tabla 1. Especies de Euchromiina del departamento de Loreto.

\begin{tabular}{|c|c|c|}
\hline № & Especie & Número de localidad \\
\hline 1 & Pseudopompilia mimica Druce, $1898^{1}$ & $64,68,73,77,96$ \\
\hline 2 & Pseudopompilia vespa (Herrich-Schäffer, 1855) ${ }^{2}$ & 63,68 \\
\hline 3 & Orcynia calcarata (Walker, 1854) ${ }^{3}$ & $52,58,64,66,79,87,96,100$ \\
\hline 4 & Myrmecopsis polistes (Hübner, [1813]) & $63,75,79$ \\
\hline 5 & Myrmecopsis nr. pellax (Draudt, 1916) & $63,64,75,82,87,98$ \\
\hline 6 & Pleurosoma angustata (Möschler, 1877) ${ }^{5}$ & $50,63,64,75,79,87,98$ \\
\hline 7 & Sphecosoma abnormis (Hampson, 1898) & $64,68,82,87$ \\
\hline 8 & Sphecosoma nr. surrentum Druce, $1883^{7}$ & $64,75,98$ \\
\hline 9 & Sphecosoma sp. 1 & $64,68,79,98$ \\
\hline 10 & Pompiliodes aliena (Walker, 1854) & $7,8,21,29,39,40,55,63,79,81,95$ \\
\hline 11 & Methysia notabilis Walker, 1854 & $68,75,87$ \\
\hline 12 & Homoeocera nr. ianthina Draudt, 1917 & $72,79,94$ \\
\hline 13 & Isanthrene aterrima (Walker, 1854$)^{8}$ & $39,58,63,64,68,69,75,79,82,87,94,97,99$ \\
\hline 14 & Isanthrene melas (Cramer, [1775]) & $62,68,79,94,96$ \\
\hline 15 & Isanthrene porphyrius (Walker, 1854) & $39,42,63,67,68,69,79,87,94,95,97$ \\
\hline 16 & Isanthrene profusa Hampson, $1898^{9}$ & 8 \\
\hline 17 & Isanthrene varia (Walker, 1854) & $39,69,79,82,91,95$ \\
\hline 18 & Isanthrene nr. vespiformis (Butler, 1876) & 39,95 \\
\hline 19 & Hyda basilutea (Walker, 1854) & $25,29,46,52$ \\
\hline 20 & Autochloris caunus (Cramer, 1779) & $39,64,69,77,79,82,87,94,95,97,99$ \\
\hline 21 & Autochloris collocata (Walker, [1865] $)^{10}$ & $49,58,62,64,79,94,95,100$ \\
\hline 22 & Autochloris completa (Walker, 1854) & $11,16,21,30,39,42,44,50,62,64,66,68,69,78,79,82,87,94,95,99,105$ \\
\hline 23 & Autochloris consociata (Walker, [1865]) ${ }^{11}$ & $68,72,76,79,83,94$ \\
\hline 24 & Autochloris solimoes Schaus, $1924^{12}$ & $21,32,40,86,95$ \\
\hline 25 & Autochloris whiteleyi (Druce, 1883)* & Este de Perú \\
\hline 26 & Sarosa acutior (Felder, 1874) & $15,21,36,39,40,49,61,66,68,72,77,79,87,93,95,96,99,100,102,105$ \\
\hline 27 & Sarosa ignicornis Hampson, $1914^{13}$ & 84,90 \\
\hline 28 & Tigrinadia quadricincta Kaye, $1918^{14}$ & $39,58,64,68,79,94$ \\
\hline 29 & Andrenimorpha abdominalis (Rothschild, 1931)* & 77 \\
\hline 30 & Andrenimorpha eusebia (Druce, 1883) ${ }^{15}$ & 43,85 \\
\hline 31 & Andrenimorpha lycopolis (Druce, 1883) & 54,68 \\
\hline 32 & Andrenimorpha sephela (Druce, 1883) & $4,42,49,64,79$ \\
\hline 33 & Andrenimorpha stuarti (Rothschild, 1911)* & 69 \\
\hline 34 & Andrenimorpha villia (Druce, 1906) & $20,64,79,94,99$ \\
\hline 35 & Andrenimorpha $\mathrm{nr}$. xantogastra (Perty, 1833) & 84 \\
\hline 36 & Phoenicoprocta rubiventer amazonica Zerny, 1930 & $39,64,81,102$ \\
\hline 37 & Phoenicoprocta vacillans (Walker, 1856) & $41,45,49,51,58,64,68,69,75,78,79,82,87,94$ \\
\hline 38 & Pheia albisigna (Walker, 1854) & 11 \\
\hline 39 & Pheia gaudens (Walker, 1856) & 82 \\
\hline 40 & Pheia nanata Kaye, 1918* & Río Pacaya \\
\hline 41 & Pheia simillima Kaye, 1919* & Río Ucayali \\
\hline 42 & Pheia toulgoeti Cerda, 2008 & $49,61,73$ \\
\hline 43 & Loxophlebia chrysobasis Dognin, 1912 & 50,100 \\
\hline 44 & Loxophlebia diaphana (Sepp, 1848) & $1,8,11,21,49$ \\
\hline 45 & Loxophlebia ribripicta Dognin, 1916 & 50,100 \\
\hline 46 & Heterodontia chalonitis (Druce, 1883) & 40 \\
\hline 47 & Mesothen bisexualis Dognin, 1912 & 61,85 \\
\hline
\end{tabular}




\begin{tabular}{|c|c|c|}
\hline № & Especie & Número de localidad \\
\hline 48 & Mesothen desperata (Walker, 1856) & 58 \\
\hline 49 & Mimagyrta abdominalis (Rothschild, 1912) & 94 \\
\hline 50 & Heterodontia sp. & 49,77 \\
\hline 51 & Heterodontia decisa (Walker, [1865]) & $2,9,17,21,49,50,73,79$ \\
\hline 52 & Heterodontia fenestrina (Butler, 1876) & $7,8,9,10,12,19,21,23,31,33,49,77,100$ \\
\hline 53 & Nyrydela chalciope (Hübner, [1831]) & $40,58,64,68,73,79,87,94$ \\
\hline 54 & Leucotmemis nr. hemileuca (Butler, 1876) & 103 \\
\hline 55 & Leucotmemis nr. leucotmemica (Bryk, 1953) ${ }^{17}$ & $39,49,50,53,54,61,77,79,89,101,103$ \\
\hline 56 & Leucotmemis melini Bryk, 1953* & Este de Perú \\
\hline 57 & Leucotmemis nexa (Herrich-Schäffer, [1854]) & $58,60,64,77,79,87,94$ \\
\hline 58 & Leucotmemis tenthredoides (Walker, 1856) & $6,16,39,50,61,94,96,97,100$ \\
\hline 59 & Leucotmemis torrida (Walker, $1854^{1} 8$ & $15,18,39,63,73,79,96$ \\
\hline 60 & Leucotmemis sp. 1 & $1,11,21,27$ \\
\hline 61 & Leucotmemis sp. 2 & $1,8,9,11,13,23,24,33,95$ \\
\hline 62 & Cosmosoma contracta (Walker, 1856) ${ }^{19}$ & $60,68,79,94$ \\
\hline 63 & Cosmosoma corvica Dognin, 1911 & 77,95 \\
\hline 64 & Cosmosoma nr. galatea Schaus, 1912 & $5,40,94,95$ \\
\hline 65 & Cosmosoma ochreipennis Kaye, 1918* & 62 \\
\hline 66 & Cosmosoma phoenicophora Dognin, 1909 & $7,8,9,19,21,29,30,32$ \\
\hline 67 & Cosmosoma regia (Schaus, 1894) & 42 \\
\hline 68 & Cosmosoma subflamma (Walker, 1854) & $58,66,68,94$ \\
\hline 69 & Cosmosoma telephus (Walker, 1854) & 39,43 \\
\hline 70 & Cosmosoma teuthras (Walker, 1854) & 64 \\
\hline 71 & Cosmosoma thoracicum Schaus, 1905 & 54 \\
\hline 72 & Cosmosoma xanthostictum Hampson, 1898 & 55 \\
\hline 73 & Cosmosoma sp. & $79,87,94$ \\
\hline 74 & Xanthida chalcosticta (Butler, 1876) & $49,50,56,64,65,68,77,85,87,94,95$ \\
\hline 75 & Mystrocneme varipes (Walker, 1854) 20 & 41,64 \\
\hline 76 & Dixophlebia quadristrigata (Walker, [1865)] & $1,21,64,82,98$ \\
\hline 77 & Pseudocharis nigrozonum Schaus, 1905 & $32,39,49,73,81$ \\
\hline 78 & Pseudocharis sp. & $10,12,14,39$ \\
\hline 79 & Hypocharis clusia (Druce, 1897) & $1,3,7,9,11,12,13,16,21,22,23,24,25,27,34,35,37,38,49,95$ \\
\hline 80 & Pseudomya biradiata (Felder, 1874) & 3 \\
\hline 81 & Pseudomya cassandra (Linnaeus, 1758) & $1,21,39,40,49,50,58,71,73,77,79,81,94,95$ \\
\hline 82 & Pseumodya cretheis (Druce, 1883) ${ }^{22}$ & $40,53,69,102$ \\
\hline 83 & Pseudomya nr. fumosa (Schaus, 1914) & $9,12,16,21,37,43,61$ \\
\hline 84 & Pseudomya hilda (Druce, 1906) ${ }^{23}$ & 1,28 \\
\hline 85 & Pseudomya lacteata (Butler, 1877) & $13,30,33,90$ \\
\hline 86 & Pseudomya lasiphlebia (Dognin, 1906) & $39,49,95$ \\
\hline 87 & Pseudomya pebasa (Kaye, 1918) & $1,7,8,9,19,31,32,35,40,95$ \\
\hline 88 & Pseudomya nr. phoenicosticta (Hampson, 1898) & 9,95 \\
\hline 89 & Pseudomya temenus (Stoll, [1781]) & $1,5,8,9,10,11,18,21,23,26,29,32,49,50,63,68,74,77,82,87,95$ \\
\hline 90 & Pseudomya tipulina (Hübner, [1812]) & $40,68,70,87,95$ \\
\hline 91 & Pseudomya vindonissa (Druce, 1883) ${ }^{24}$ & $21,39,49,50,51,66,77,79$ \\
\hline 92 & Pseudomya sp. & 49,103 \\
\hline 93 & Micragyrta diminuta (Walker, 1854) & 41,95 \\
\hline 94 & Psoloptera thoracica (Walker, 1854) & $21,36,49,68,79,80,83,96,100$ \\
\hline 95 & Histioea amazonica Butler, 1876 & 77,79 \\
\hline 96 & Histioea bellatrix (Walker, 1854) & 77 \\
\hline 97 & Histioea imaon Hampson, 1898 & 59,105 \\
\hline 98 & Histioea paulina Walker, 1866 & 48 \\
\hline 99 & Histioea proserpina (Hübner, 1823) & 69,79 \\
\hline 100 & Histioea ramirezi Grados, MS & Loreto \\
\hline 101 & Bodosa tina (Walker, 1854) & $41,69,76,83,88$ \\
\hline 102 & Agyrtidia uranophila (Walker, 1866) & $57,62,67,69,70,92,104$ \\
\hline 103 & Agyrtiola niepelti Gaede, 1926 & 94 \\
\hline 104 & Macrocneme bodoquero Dietz, 1994* & 77 \\
\hline 105 & Macrocneme durcata Dietz, 1994* & Pumayacu \\
\hline 106 & Macrocneme imbelis Sietz, 1994* & 69 \\
\hline 107 & Macrocneme orichalcea Dietz, 1994* & Río Marañón \\
\hline 108 & Calonotos aequimaculatus Zerny, 1931 & 81,94 \\
\hline 109 & Calonotos tiburtus (Cramer, [1779]) & 41 \\
\hline
\end{tabular}




\begin{tabular}{lll}
\hline No & Especie & Número de localidad \\
\hline 110 & Calonotos longipennis Rothschild, 1911 & 39,94 \\
111 & Poliopastea anthracina (Klages, 1906) & 41 \\
112 & Poliopastea indistincta (Butler, 1876) & 47 \\
113 & Poliopastea plumbea Hampson, 1898 & 95 \\
\hline
\end{tabular}

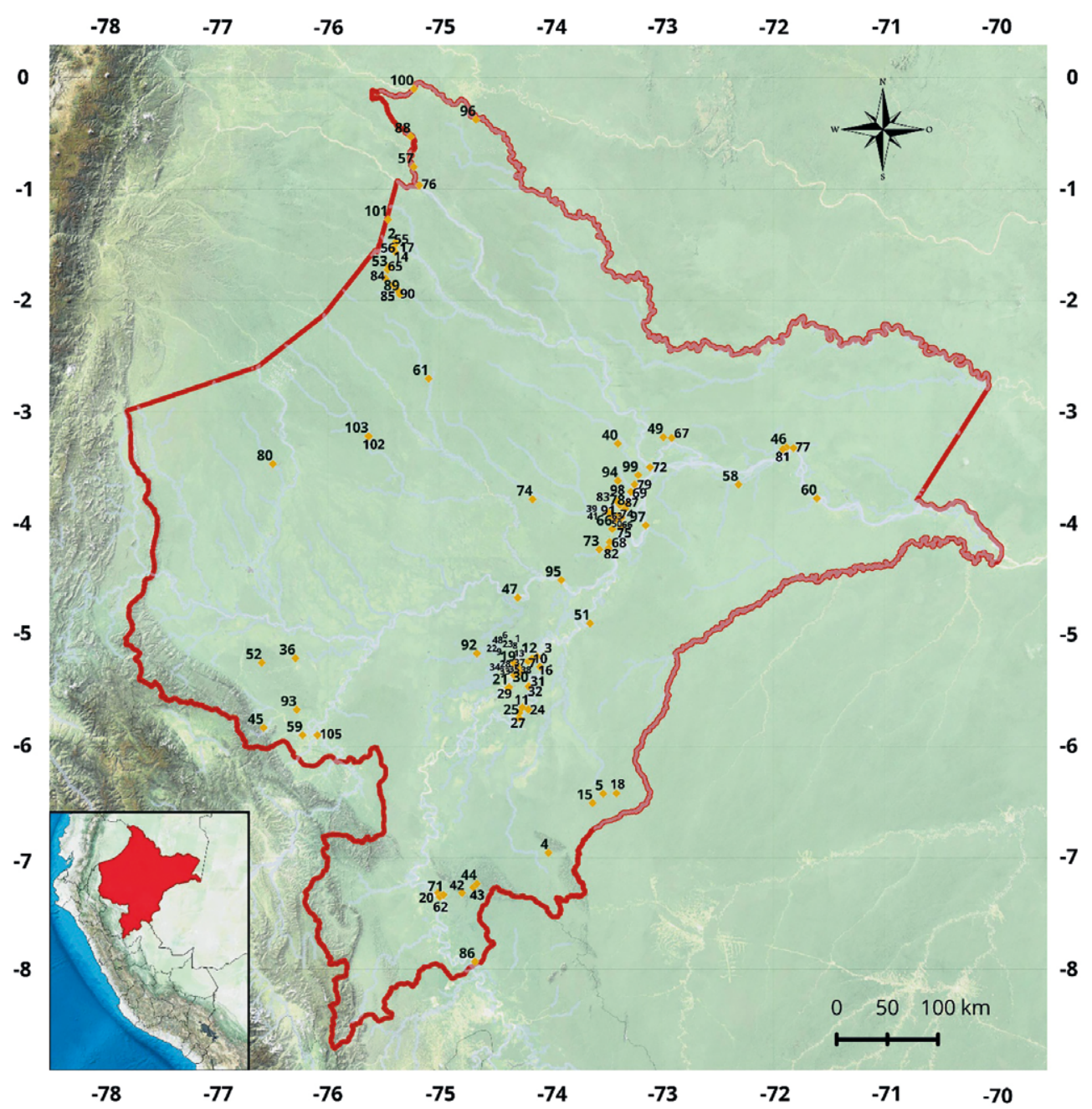

Figura 1. Mapa de Loreto mostrando los lugares de recolecta de Euchromiina "polillas avispas" (Lepidoptera: Erebidae: Arctiinae: Arctiini).

Tabla 2. Localidades de recolecta de los Euchromiina en Loreto.

\begin{tabular}{cllll}
\hline No de localidad & Nombre de la localidad & Altura & Latitud & Longitud \\
\hline 1 & $1.83 \mathrm{~km}$ ENE de Bretaña & $107 \mathrm{~m}$ & $05^{\circ} 14^{\prime} 48^{\prime \prime}$ & $74^{\circ} 19^{\prime} 45^{\prime \prime}$ \\
2 & $10.5 \mathrm{~km}$ NO de El Pedregal & $164 \mathrm{~m}$ & $01^{\circ} 28^{\prime} 34^{\prime \prime}$ & $75^{\circ} 23^{\prime} 46^{\prime \prime}$ \\
3 & $11.5 \mathrm{~km}$ NO de Huatape & $111 \mathrm{~m}$ & $05^{\circ} 12^{\prime} 50^{\prime \prime}$ & $7^{\circ} 09^{\prime} 51^{\prime \prime}$ \\
4 & $12.5 \mathrm{~km}$ E Pto. Venus & $225 \mathrm{~m}$ & $06^{\circ} 57^{\prime} 21^{\prime \prime}$ & $74^{\circ} 01^{\prime} 27^{\prime \prime}$ \\
5 & $14 \mathrm{~km}$ SE de Qda. Lobo & $174 \mathrm{~m}$ & $06^{\circ} 25^{\prime} 31^{\prime \prime}$ & $73^{\circ} 31^{\prime} 54^{\prime \prime}$ \\
6 & $15 \mathrm{~km}$ lquitos-Nauta & $120 \mathrm{~m}$ & $03^{\circ} 57^{\prime}$ & $73^{\circ} 22^{\prime}$ \\
7 & $16 \mathrm{~km}$ NE de Bretaña, Canal de Puinahua & $113 \mathrm{~m}$ & $05^{\circ} 14^{\prime} 15^{\prime \prime}$ & $74^{\circ} 12^{\prime} 22^{\prime \prime}$ \\
8 & $2.37 \mathrm{~km}$ ENE de Bretaña & $110 \mathrm{~m}$ & $05^{\circ} 14^{\prime} 58^{\prime \prime}$ & $74^{\circ} 19^{\prime} 22^{\prime \prime}$ \\
9 & $2.47 \mathrm{~km}$ ENE de Bretaña & $115 \mathrm{~m}$ & $05^{\circ} 15^{\prime} 02^{\prime \prime}$ & $74^{\circ} 19^{\prime} 19^{\prime \prime}$ \\
10 & $20 \mathrm{~km}$ NE de Bretaña, Canal de Puinahua & $104 \mathrm{~m}$ & $05^{\circ} 12^{\prime} 59^{\prime \prime}$ & $74^{\circ} 10^{\prime} 27^{\prime \prime}$ \\
11 & $3 \mathrm{~km}$ NO de Palizada & $115 \mathrm{~m}$ & $05^{\circ} 39^{\prime} 04^{\prime \prime}$ & $74^{\circ} 15^{\prime} 28^{\prime \prime}$ \\
12 & $3 \mathrm{~km} \mathrm{SO}$ de Zapote, río Ucayali & $123 \mathrm{~m}$ & $05^{\circ} 11^{\prime} 43^{\prime \prime}$ & $74^{\circ} 07^{\prime} 31^{\prime \prime}$ \\
13 & $3.2 \mathrm{~km}$ NE de Bretaña & $105 \mathrm{~m}$ & $05^{\circ} 15^{\prime} 57^{\prime \prime}$ & $74^{\circ} 19^{\prime} 30^{\prime \prime}$ \\
14 & $3.3 \mathrm{~km}$ E de El Pedregal & $162 \mathrm{~m}$ & $01^{\circ} 33^{\prime} 44^{\prime \prime}$ & $75^{\circ} 24^{\prime} 03^{\prime \prime}$ \\
15 & $3.5 \mathrm{~km}$ E de Qda. Lobo & $167 \mathrm{~m}$ & $06^{\circ} 30^{\prime} 34^{\prime \prime}$ & $73^{\circ} 37^{\prime} 37^{\prime \prime}$
\end{tabular}




\begin{tabular}{|c|c|c|c|c|}
\hline № de localidad & Nombre de la localidad & Altura & Latitud & Longitud \\
\hline 17 & 3.9 km NE de El Pedregal & $153 \mathrm{~m}$ & $01^{\circ} 32^{\prime} 12^{\prime \prime}$ & $75^{\circ} 25^{\prime} 10^{\prime \prime}$ \\
\hline 18 & 4 km S de Betilia & $159 \mathrm{~m}$ & $06^{\circ} 25^{\prime} 22^{\prime \prime}$ & $73^{\circ} 24^{\prime} 47^{\prime \prime}$ \\
\hline 19 & $4.3 \mathrm{~km}$ NE de Bretaña & $122 \mathrm{~m}$ & $05^{\circ} 16^{\prime} 11^{\prime \prime}$ & $74^{\circ} 18^{\prime} 57^{\prime \prime}$ \\
\hline 20 & $5 \mathrm{~km}$ NNW de Contamana & $180 \mathrm{~m}$ & $07^{\circ} 19^{\prime}$ & $75^{\circ} 01^{\prime}$ \\
\hline 21 & 5 km NO de La Palma & $115 \mathrm{~m}$ & $05^{\circ} 28^{\prime} 28^{\prime \prime}$ & $74^{\circ} 23^{\prime} 04^{\prime \prime}$ \\
\hline 22 & 5.3 km NNE de Bretaña & $120 \mathrm{~m}$ & $05^{\circ} 14^{\prime} 47^{\prime \prime}$ & $74^{\circ} 19^{\prime} 35^{\prime \prime}$ \\
\hline 23 & 5.4 km NNE de Bretaña & $103 \mathrm{~m}$ & $05^{\circ} 14^{\prime} 37^{\prime \prime}$ & $74^{\circ} 19^{\prime} 26^{\prime \prime}$ \\
\hline 24 & 5.5 km ENE de Palizada & $108 \mathrm{~m}$ & $05^{\circ} 40^{\prime} 08^{\prime \prime}$ & $74^{\circ} 12^{\prime} 16^{\prime \prime}$ \\
\hline 25 & $5.5 \mathrm{~km} \mathrm{~N}$ de Tamanco & $112 \mathrm{~m}$ & $05^{\circ} 44^{\prime} 51^{\prime \prime}$ & $74^{\circ} 17^{\prime} 13^{\prime \prime}$ \\
\hline 26 & 5.5 km NNE de Bretaña & $120 \mathrm{~m}$ & $05^{\circ} 14^{\prime} 47^{\prime \prime}$ & $74^{\circ} 19^{\prime} 35^{\prime \prime}$ \\
\hline 27 & $5.5 \mathrm{~km}$ SO de Palizada & $115 \mathrm{~m}$ & $05^{\circ} 42^{\prime} 03^{\prime \prime}$ & $74^{\circ} 17^{\prime} 12^{\prime \prime}$ \\
\hline 28 & 6.35 km ESE de Bretaña & $114 \mathrm{~m}$ & $05^{\circ} 18^{\prime} 02^{\prime \prime}$ & $74^{\circ} 17^{\prime} 32^{\prime \prime}$ \\
\hline 29 & 7 km NO de La Palma & $113 \mathrm{~m}$ & $05^{\circ} 28^{\prime} 10^{\prime \prime}$ & $74^{\circ} 22^{\prime} 35^{\prime \prime}$ \\
\hline 30 & 7 km SO Flor de Punga & $127 \mathrm{~m}$ & $05^{\circ} 27^{\prime} 20^{\prime \prime}$ & $74^{\circ} 12^{\prime} 08^{\prime \prime}$ \\
\hline 31 & 7.5 km SO de Flor de Punga & $104 \mathrm{~m}$ & $05^{\circ} 27^{\prime} 28^{\prime \prime}$ & $74^{\circ} 12^{\prime} 08^{\prime \prime}$ \\
\hline 32 & $8 \mathrm{~km}$ SO de Flor de Punga & $103 \mathrm{~m}$ & $05^{\circ} 27^{\prime} 46^{\prime \prime}$ & $74^{\circ} 12^{\prime} 09^{\prime \prime}$ \\
\hline 33 & 8 km SSE de Bretaña & $103 \mathrm{~m}$ & $05^{\circ} 21^{\prime} 43^{\prime \prime}$ & $74^{\circ} 19^{\prime} 54^{\prime \prime}$ \\
\hline 34 & 8.5 km SSE de Bretaña & $108 \mathrm{~m}$ & $05^{\circ} 21^{\prime} 46^{\prime \prime}$ & $74^{\circ} 19^{\prime} 52^{\prime \prime}$ \\
\hline 35 & 8.8 km SE de Bretaña & $90 \mathrm{~m}$ & $05^{\circ} 20^{\prime} 10^{\prime \prime}$ & $74^{\circ} 16^{\prime} 59^{\prime \prime}$ \\
\hline 36 & $9 \mathrm{~km} \mathrm{~N}$ de Jeberos & $121 \mathrm{~m}$ & $05^{\circ} 12^{\prime} 34^{\prime \prime}$ & $76^{\circ} 17^{\prime} 51^{\prime \prime}$ \\
\hline 37 & 9.5 km SE de Bretaña & $113 \mathrm{~m}$ & $05^{\circ} 20^{\prime} 09^{\prime \prime}$ & $74^{\circ} 16^{\prime} 37^{\prime \prime}$ \\
\hline 38 & 9.9 km SE de Bretaña & $96 \mathrm{~m}$ & $05^{\circ} 20^{\prime} 30^{\prime \prime}$ & $74^{\circ} 16^{\prime} 49^{\prime \prime}$ \\
\hline 39 & Agua Blanca & $130 \mathrm{~m}$ & $03^{\circ} 56^{\prime}$ & $73^{\circ} 28^{\prime}$ \\
\hline 40 & Allpahuayo-Mishana 1 & $150 \mathrm{~m}$ & $03^{\circ} 17^{\prime}$ & $73^{\circ} 24^{\prime}$ \\
\hline 41 & Allpahuayo-Mishana 2 & $130 \mathrm{~m}$ & $03^{\circ} 56^{\prime}-59^{\prime}$ & $73^{\circ} 24^{\prime}-25^{\prime}$ \\
\hline 42 & Alto río Contaya, Campamento I & $400 \mathrm{~m}$ & $07^{\circ} 19^{\prime}$ & $74^{\circ} 48^{\prime}$ \\
\hline 43 & Alto río Contaya, Campamento II & $250 \mathrm{~m}$ & $07^{\circ} 16^{\prime}$ & $74^{\circ} 42^{\prime}$ \\
\hline 44 & Alto río Contaya, Campamento III & $250 \mathrm{~m}$ & $07^{\circ} 14^{\prime}$ & $74^{\circ} 40^{\prime}$ \\
\hline 45 & Balsapuerto & $200 \mathrm{~m}$ & $05^{\circ} 50^{\prime}$ & $76^{\circ} 35^{\prime}$ \\
\hline 46 & Betania & $120 \mathrm{~m}$ & $03^{\circ} 19^{\prime}$ & $71^{\circ} 53^{\prime}$ \\
\hline 47 & Boca del Rio Samiria & $120 \mathrm{~m}$ & $04^{\circ} 40^{\prime}$ & $74^{\circ} 18^{\prime}$ \\
\hline 48 & Bretaña & $100 \mathrm{~m}$ & $05^{\circ} 15^{\prime}$ & $74^{\circ} 20^{\prime}$ \\
\hline 49 & Brillo Nuevo & $90 \mathrm{~m}$ & $03^{\circ} 13^{\prime} 35^{\prime \prime}$ & $72^{\circ} 59^{\prime} 30^{\prime \prime}$ \\
\hline 50 & C.I. Allpahuayo-Mishana & $118 \mathrm{~m}$ & $03^{\circ} 58^{\prime} 01^{\prime \prime}$ & $73^{\circ} 25^{\prime} 08^{\prime \prime}$ \\
\hline 51 & C.I. Jenaro Herrera & $125 \mathrm{~m}$ & $04^{\circ} 53^{\prime} 50^{\prime \prime}$ & $73^{\circ} 39^{\prime} 00^{\prime \prime}$ \\
\hline 52 & ca. Lagunas & $136 \mathrm{~m}$ & $05^{\circ} 15^{\prime}$ & $76^{\circ} 36^{\prime}$ \\
\hline 53 & Campamento Dorado 4, río Curaray & $218 \mathrm{~m}$ & $01^{\circ} 43^{\prime} 18^{\prime \prime}$ & $75^{\circ} 28^{\prime} 07^{\prime \prime}$ \\
\hline 54 & Campamento Dorado 5, Qda. Rumiyacu & $230 \mathrm{~m}$ & $01^{\circ} 48^{\prime} 09^{\prime \prime}$ & $75^{\circ} 29^{\prime} 14^{\prime \prime}$ \\
\hline 55 & Campamento Paiche, río Curaray 1 & $200 \mathrm{~m}$ & $01^{\circ} 29^{\prime} 25^{\prime \prime}$ & $75^{\circ} 23^{\prime} 53^{\prime \prime}$ \\
\hline 56 & Campamento Paiche, río Curaray 2 & $215 \mathrm{~m}$ & $01^{\circ} 32^{\prime} 00^{\prime \prime}$ & $75^{\circ} 25^{\prime} 16^{\prime \prime}$ \\
\hline 57 & Castaña & $150 \mathrm{~m}$ & $00^{\circ} 48^{\prime}$ & $75^{\circ} 14^{\prime}$ \\
\hline 58 & Centro Fuerte & $120 \mathrm{~m}$ & $03^{\circ} 39^{\prime}$ & $72^{\circ} 19^{\prime}$ \\
\hline 59 & Chambira, río Parapanura & $120 \mathrm{~m}$ & $05^{\circ} 54^{\prime}$ & $76^{\circ} 14^{\prime}$ \\
\hline 60 & Cochiquinas & $90 \mathrm{~m}$ & $03^{\circ} 46^{\prime} 30^{\prime \prime}$ & $71^{\circ} 36^{\prime} 40^{\prime \prime}$ \\
\hline 61 & Coconilla & $160 \mathrm{~m}$ & $02^{\circ} 42^{\prime}$ & $75^{\circ} 06^{\prime}$ \\
\hline 62 & Contamana & $150 \mathrm{~m}$ & $07^{\circ} 21^{\prime}$ & $75^{\circ} 00^{\prime}$ \\
\hline 63 & El Dorado & $136 \mathrm{~m}$ & $03^{\circ} 57^{\prime} 13^{\prime \prime}$ & $73^{\circ} 24^{\prime} 28^{\prime \prime}$ \\
\hline 64 & El Milagro & $120 \mathrm{~m}$ & $03^{\circ} 57^{\prime}$ & $73^{\circ} 22^{\prime}$ \\
\hline 65 & Entre río Arabela y río Curaray & $240 \mathrm{~m}$ & $01^{\circ} 41^{\prime} 52^{\prime \prime}$ & $75^{\circ} 27^{\prime} 23^{\prime \prime}$ \\
\hline 66 & Ex-Petroleros, km 46 Carr. Iquitos-Nauta & $160 \mathrm{~m}$ & $04^{\circ} 03^{\prime}$ & $73^{\circ} 27^{\prime}$ \\
\hline 67 & Explornapo-ACEER, Río Sucusari & $140 \mathrm{~m}$ & $03^{\circ} 14^{\prime}$ & $72^{\circ} 55^{\prime}$ \\
\hline 68 & Huambe & $112 \mathrm{~m}$ & $04^{\circ} 10^{\prime} 05^{\prime \prime}$ & $73^{\circ} 28^{\prime} 27^{\prime \prime}$ \\
\hline 69 & Iquitos & $100 \mathrm{~m}$ & $03^{\circ} 43^{\prime}$ & $73^{\circ} 17^{\prime}$ \\
\hline 70 & km 15 Iquitos-Nauta & $120 \mathrm{~m}$ & $03^{\circ} 57^{\prime}$ & $73^{\circ} 22^{\prime}$ \\
\hline 71 & Maquía & $185 \mathrm{~m}$ & $07^{\circ} 20^{\prime}$ & $74^{\circ} 58^{\prime}$ \\
\hline 72 & Mazán & $100 \mathrm{~m}$ & $03^{\circ} 29^{\prime} 45^{\prime \prime}$ & $73^{\circ} 06^{\prime} 40^{\prime \prime}$ \\
\hline 73 & Melitón Carbajal & $153 \mathrm{~m}$ & $04^{\circ} 14^{\prime}$ & $73^{\circ} 34^{\prime}$ \\
\hline 74 & Padre Isla & $120 \mathrm{~m}$ & $03^{\circ} 47^{\prime}$ & $74^{\circ} 10^{\prime}$ \\
\hline 75 & Palo seco & $100 \mathrm{~m}$ & $04^{\circ} 02^{\prime}$ & $73^{\circ} 22^{\prime}$ \\
\hline 76 & Pantoja & $180 \mathrm{~m}$ & $00^{\circ} 58^{\prime}$ & $75^{\circ} 11^{\prime}$ \\
\hline 77 & Pebas & $80 \mathrm{~m}$ & $03^{\circ} 19^{\prime} 24^{\prime \prime}$ & $71^{\circ} 49^{\prime} 17^{\prime \prime}$ \\
\hline 78 & Peña Negra & $130 \mathrm{~m}$ & $03^{\circ} 52^{\prime}$ & $73^{\circ} 20^{\prime}$ \\
\hline
\end{tabular}




\begin{tabular}{|c|c|c|c|c|}
\hline № de localidad & Nombre de la localidad & Altura & Latitud & Longitud \\
\hline 79 & Picuroyacu & $110 \mathrm{~m}$ & $03^{\circ} 39^{\prime}$ & $73^{\circ} 15^{\prime}$ \\
\hline 80 & Pucacuro & $170 \mathrm{~m}$ & $03^{\circ} 28^{\prime}$ & $76^{\circ} 30^{\prime}$ \\
\hline 81 & Pucaurquillo & $120 \mathrm{~m}$ & $03^{\circ} 20^{\prime}$ & $71^{\circ} 55^{\prime}$ \\
\hline 82 & Puente Itaya & $99 \mathrm{~m}$ & $04^{\circ} 13^{\prime} 20^{\prime \prime}$ & $73^{\circ} 29^{\prime} 00^{\prime \prime}$ \\
\hline 83 & Puerto Almendra & $120 \mathrm{~m}$ & $03^{\circ} 50^{\prime}$ & $73^{\circ} 23^{\prime}$ \\
\hline 84 & Qda. Rumiyacu, Campamento Dorado 5 & $230 \mathrm{~m}$ & $01^{\circ} 48^{\prime} 09^{\prime \prime}$ & $75^{\circ} 29^{\prime} 14^{\prime \prime}$ \\
\hline 85 & Qda. Rumiyacu, Campamento Piraña 6 & $260 \mathrm{~m}$ & $01^{\circ} 51^{\prime} 29^{\prime \prime}$ & $75^{\circ} 25^{\prime} 19^{\prime \prime}$ \\
\hline 86 & Quebrada Tunuya & $130 \mathrm{~m}$ & $07^{\circ} 56^{\prime}$ & $74^{\circ} 41^{\prime}$ \\
\hline 87 & Quistococha, km 6 Carr. Iquitos-Nauta & $100 \mathrm{~m}$ & $03^{\circ} 49^{\prime} 30^{\prime \prime}$ & $73^{\circ} 19^{\prime} 16^{\prime \prime}$ \\
\hline 88 & Río Aguas Negras & $150 \mathrm{~m}$ & $00^{\circ} 31^{\prime} 38^{\prime \prime}$ & $75^{\circ} 15^{\prime} 41^{\prime \prime}$ \\
\hline 89 & Río Arabela, Campamento Piraña 7A & $221 \mathrm{~m}$ & $01^{\circ} 56^{\prime} 28^{\prime \prime}$ & $75^{\circ} 21^{\prime} 38^{\prime \prime}$ \\
\hline 90 & Río Arabela, Campamento Piraña 7B & $190 \mathrm{~m}$ & $01^{\circ} 54^{\prime} 06^{\prime \prime}$ & $75^{\circ} 22^{\prime} 56^{\prime \prime}$ \\
\hline 91 & Est. Biol. Callicebus, Río Nanay & $150 \mathrm{~m}$ & $03^{\circ} 54^{\prime}$ & $73^{\circ} 29^{\prime}$ \\
\hline 92 & Est. Biológica Pithecia, Río Samiria & $180 \mathrm{~m}$ & $05^{\circ} 10^{\prime}$ & $74^{\circ} 40^{\prime}$ \\
\hline 93 & Río Zapote & $173 \mathrm{~m}$ & $05^{\circ} 40^{\prime} 15^{\prime \prime}$ & $76^{\circ} 17^{\prime} 08^{\prime \prime}$ \\
\hline 94 & San Juan de Poli & $120 m$ & $03^{\circ} 37^{\prime}$ & $73^{\circ} 24^{\prime}$ \\
\hline 95 & San Regis (Albergue La Posada) & $130 \mathrm{~m}$ & $04^{\circ} 30^{\prime} 30^{\prime \prime}$ & $73^{\circ} 54^{\prime} 30^{\prime \prime}$ \\
\hline 96 & Soplín Vargas & $210 \mathrm{~m}$ & $00^{\circ} 22^{\prime} 30^{\prime \prime}$ & $74^{\circ} 40^{\prime} 30^{\prime \prime}$ \\
\hline 97 & Tamshiyacu & $100 \mathrm{~m}$ & $04^{\circ} 01^{\prime}$ & $73^{\circ} 09^{\prime}$ \\
\hline 98 & Tarapoto, río Nanay & $120 m$ & $03^{\circ} 48^{\prime} 10^{\prime \prime}$ & $73^{\circ} 24^{\prime} 20^{\prime \prime}$ \\
\hline 99 & Tierra Hermosa & $140 \mathrm{~m}$ & $03^{\circ} 34^{\prime}$ & $73^{\circ} 13^{\prime}$ \\
\hline 100 & Tres Fronteras & $220 \mathrm{~m}$ & $00^{\circ} 06^{\prime} 00^{\prime \prime}$ & $75^{\circ} 14^{\prime} 00^{\prime \prime}$ \\
\hline 101 & Trompeteros 1 & $151 \mathrm{~m}$ & $01^{\circ} 16^{\prime} 21^{\prime \prime}$ & $75^{\circ} 27^{\prime} 54^{\prime \prime}$ \\
\hline 102 & Trompeteros 2 & $148 m$ & $03^{\circ} 13^{\prime} 23^{\prime \prime}$ & $75^{\circ} 38^{\prime} 27^{\prime \prime}$ \\
\hline 103 & Trompeteros 3 & $140 \mathrm{~m}$ & $03^{\circ} 12^{\prime} 56^{\prime \prime}$ & $75^{\circ} 38^{\prime} 26^{\prime \prime}$ \\
\hline 104 & Yanamono, $80 \mathrm{Km} \mathrm{E}$ Iquitos & $120 \mathrm{~m}$ & ca. $03^{\circ} 22^{\prime}$ & $72^{\circ} 45^{\prime}$ \\
\hline 105 & Yurimaguas & $150 \mathrm{~m}$ & $05^{\circ} 54^{\prime}$ & $76^{\circ} 06^{\prime}$ \\
\hline
\end{tabular}

\section{Notas}

1. El género Pseudopompilia Druce fue considerado en el orden filético por Hampson (1898) y Draudt (1916 - 1919) dentro de los Ctenuchina. Ha sido transferido a los Euchromiina por Simmons \& Weller (2006).

2. Pseudopompilia vespa fue descrita en base a un número no especificado de ejemplares provenientes de Pará (Brasil). Tiene una amplia distribución en la Amazonía peruana. El holotipo por ahora no ha sido ubicado. No es muy común en colecciones, quizás debido a que no son atraídas comúnmente a las trampas de luz. Los ejemplares que registramos en este trabajo han sido capturados con plantas de Heliotropium sp.

3. Orcynia calcarata es una especie común en la Amazonía peruana. Los machos poseen órganos androconiales en las tibias de las patas medias. Este tipo de órganos androconiales también se presentan en Pheia albisigna (Walker) y P. toulgoeti Cerda. Este podría ser un caso de convergencia evolutiva. Es una especie de hábitos nocturnos, de acuerdo con las observaciones de su comportamiento de obtención de pyrrolizidina sobre plantas de Heliotropium sp. realizadas en recolectas llevadas a cabo en el sudeste de Perú.

4. Myrmecopsis nr. pellax es similar a las especies $P$. noverca Schaus, $P$. novercida Kaye, $P$. polybia Kaye, P. polybioides Burmeister y P. pellax Draudt. Las tres primeras especies tienen como localidades tipo Paraná (Brasil) y los caracteres morfológicos externos consignados en las descripciones originales no ayudan mucho en la discriminación. Es necesario corroborar si en realidad se tratan de tres especies diferentes, con los caracteres de morfología de las genitalias. La cuarta especie, $P$. polybiodes fue descrita de Buenos Aires (Argentina) y es similar a las tres especies aludidas. Es probable que pueda ser sinónimo de alguna de las tres especies que ocurren en Paraná. La especie P. pellax fue descrita por Draudt (1916) con material recolectado por Bang-Haas en la región del Amazonas. Lamentablemente no se especificó el lugar exacto. Los ejemplares de Loreto son muy similares al holotipo de P. pellax, depositado en el MNHP. Sin embargo, es necesario conocer los caracteres de la morfología de la genitalia, para estar seguros que corresponde a P. pellax.

5. Pleurosoma angustata es una de las especies que son muy semejantes con avispas, y es posible que esté comprometida en complejos miméticos. El parecido morfológico no solo está limitado al cuerpo, también a la forma de las alas, el hábito diurno y la forma de vuelo. Por la presencia de estos caracteres son fácilmente confundibles. Dentro de los Euchromiina, la presencia de los órganos androconiales más comunes son los bolsillos subabdominales ("valvas ventrales"), invaginaciones en el segundo y cuarto sternito del abdomen (Hampson 1898, Barth 1953, Weller et al. 2000). Contrariamente, el órgano androconial ubicado en la parte ventral de los últimos segmentos abdominales, conocido como coremata, es común dentro de los Ctenuchina. Son pocos los registros de coremata dentro de los Euchromiina. Junto con Sphecosoma ecuadora Druce (Genitalia \# 248 JGA, MUSM), P. angustatum (Möschler) (Genitalia \# 168 JGA, MUSM) es una de las pocas especies dentro de los Eu- 
chromiinae que presentan coremata. Se conoce además que otras cuatro especies de Pleurosoma Orfila presentan este órgano androconial (Simons \& Weller 2006). Sin embargo, se tiene registros que al menos dos especies del género Cosmosoma Hübner presentan coremata (Grados datos inéditos).

6. Sphecosoma abnormis (Figs. 2-5) = Diptilon aurantiipes Rothschild, 1911. Nuevo sinónimo. Especie no muy común en colecciones. Es una especie de hábitos diurnos, recolectada con plantas de Heliotropium sp. Por ahora, no se ha recolectado ejemplares con trampas de luz. Hampson (1898) describió la especie basado en un ejemplar hembra (holotipo) de Massauary (bajo Amazonas, Brasil) de la colección Staudinger (ZMHB); en la misma publicación presentó un dibujo del macho. Existe un ejemplar con etiqueta del tipo en el NHMUK, siendo un error de etiquetado. Orfila (1935) creo el género Abnormipterus para Sphecosoma abnormis. Simmons y Weller (2006) consideraron la especie dentro del género Sphecosoma Butler. Rothschild (1911) describió la especie Diptilon aurantiipes con dos ejemplares machos: uno del río Amazonas de la colección Felder y el otro de Calama, río Madeira (Brasil), siendo este último la localidad tipo.
Los ejemplares machos de Rothschild (1911), de acuerdo con los caracteres morfológicos externos, corresponden con el macho que Hampson (1898) colocó en su publicación al describir la especie Sphecosoma abnormis. La hembra de $S$. abnormis y el macho de D. aurantiipes tienen los mismos caracteres morfológicos externos, salvo la presencia del órgano androconial en las alas de los machos: penacho de pelos por detrás de la $1 \mathrm{~A}+2 \mathrm{~A}$. Ambos sexos son atraídos por cebos de Heliotropium sp. y son simpátricos. Basados en esta evidencia, proponemos que Diptilon aurantiipes es un nuevo sinónimo de Sphecosoma abnormis. La especie es un nuevo registro para Perú y por ahora, ocurre solo en Loreto.

7. Sphecosoma nr. surrentum es una especie pequeña y que no es atraída comúnmente por la trampa de luz. Son de hábitos diurnos y atraídos por plantas de Heliotropium sp. La identificación es dudosa, debido a que el holotipo no se encuentra en buen estado y no permite observar claramente los caracteres. Es necesario hacer la disección del Holotipo (NHMUK) y comparar la morfología para tener certeza de la identificación.
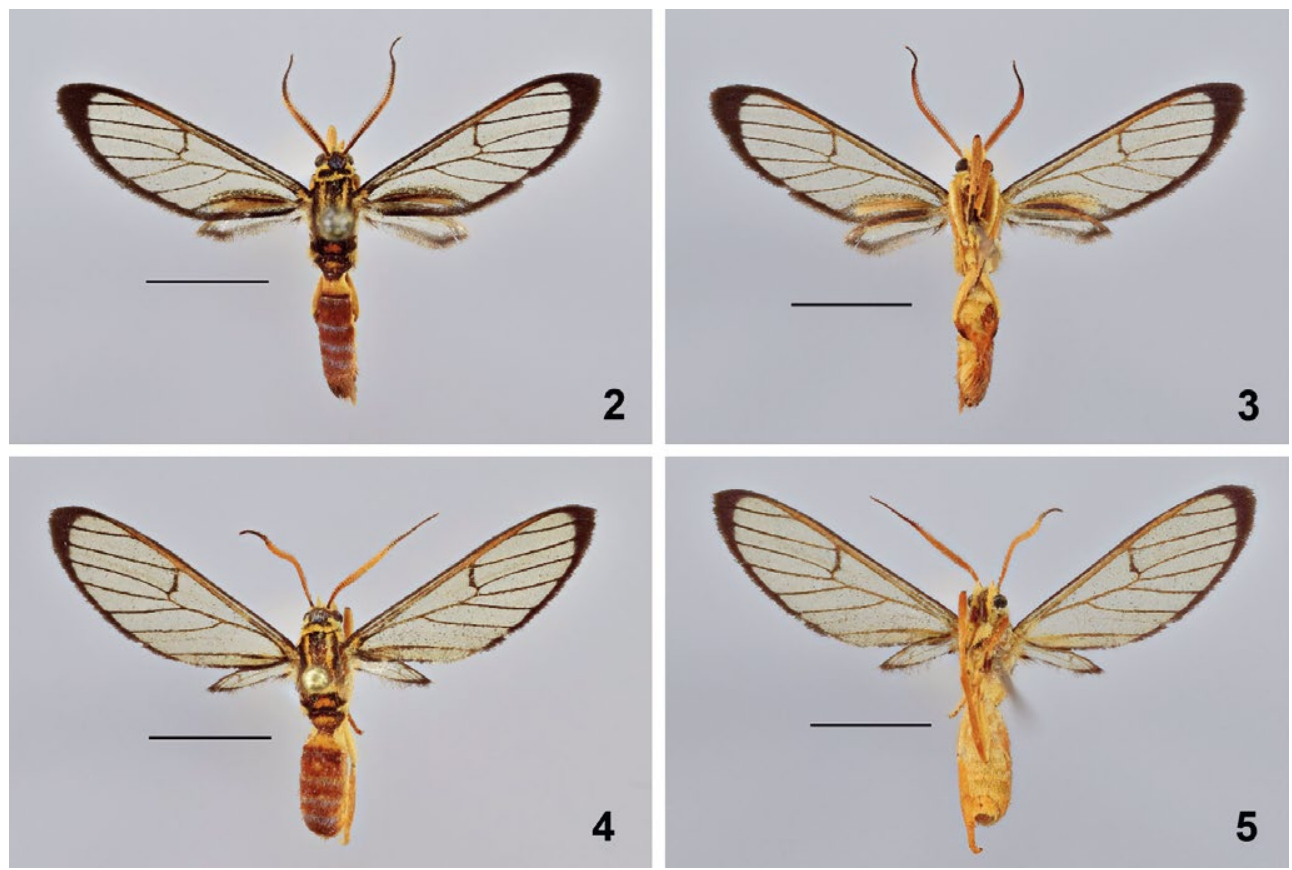

Figuras 2-5. Sphecosoma abnormis. Fig. 2-3. Macho (El Milagro). 2. Vista dorsal. 3. Vista ventral. Fig. 4-5. Hembra (Huambe). 4. Vista dorsal. 5. Vista ventral. Barra, $5 \mathrm{~mm}$.
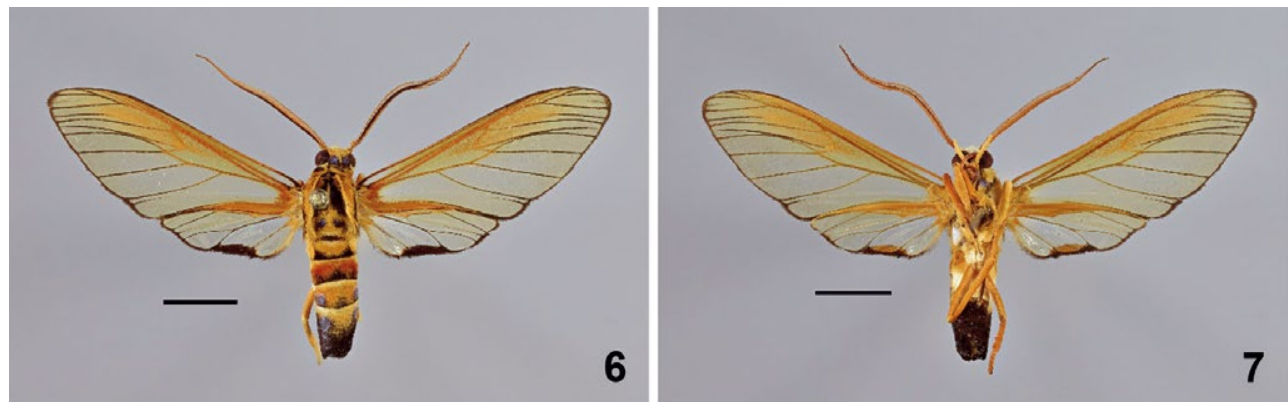

Figuras 6-7. Isanthrene profusa. Fig. 6-7. Macho (2.47 km ENE Bretaña). 7. Vista dorsal. 7. Vista ventral. Barra, $5 \mathrm{~mm}$. 
8. Isanthrene aterrima es de hábito diurno. La mayoría de los especímenes fueron recolectados con plantas de Heliotropium sp. Al parecer no son atraídos por las trampas de luz.

9. Isanthrene profusa (Figs. 6-11) fue descrita de un macho proveniente de Sao Paulo [de Olivença] (Brasil). El holotipo está depositado en el NHMUK. Es un nuevo registro para Perú.

10. Autochloris collocata (Figs. 12-13) fue descrita con un ejemplar macho proveniente de Ega (Brasil). Es un nuevo registro para el Perú y por ahora, conocida solo en Loreto.

11. Hampson (1898) consideró a Autochloris consociata (Figs. 14 - 15) como sinónimo de A. bijuncta Walker, 1856 (Figs. 16 - 17). Autochloris bijuncta fue descrita de un ejemplar hembra. En la descripción original se menciona que la especie ocurre en Pará (Brasil) y en el valle del Amazonas (Walker 1856). El holotipo está depositado en la UMO. Por otro lado, con un número no determinado de ejemplares, $A$. consociata fue descrita de Ega (Brasil). Solo se menciona en su descripción "macho". En el NHMUK existen dos ejemplares hembras etiquetados como tipos de Ega. Las hembras de las dos especies son muy similares y fácilmente confundibles. Sin embargo, en un análisis en detalle se observan diferencias: en la parte distal del abdomen, en $A$. bijuncta el área comprende los tres últimos segmentos, en tanto en $A$. consociata abarca los dos últimos; la primera especie presenta una mancha blanca en los lados del primer tergite, mientras la segunda especie no presenta. Las diferencias en la morfología de las genitalias de los machos confirman la validación de las dos especies (Figs. 18 - 25), confundidas como una sola por más de 120 años. Autochloris consociata es un nuevo registro para Perú y por ahora conocida solo de Loreto.
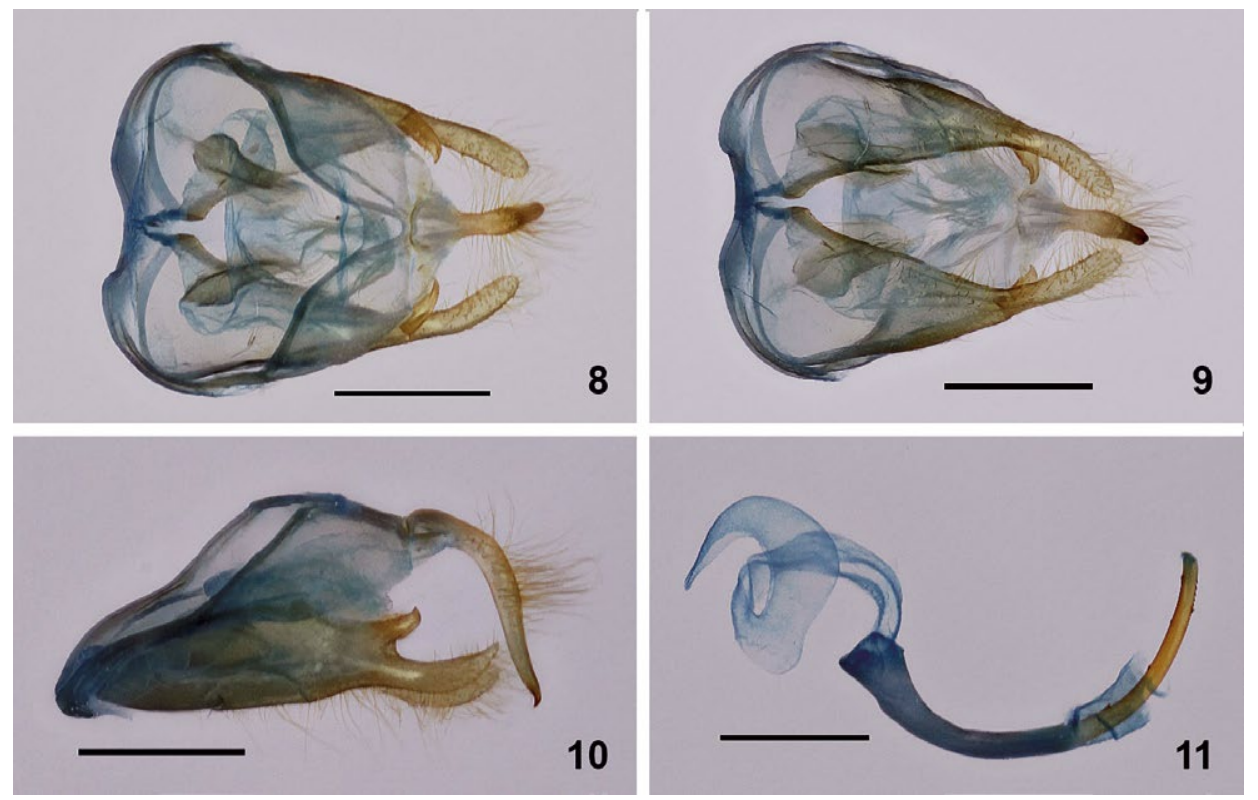

Figuras 8-11. Genital macho de Isanthrene profusa (Genitalia \# JGA-1105 MUSM) (2.47 km ENE Bretaña). 8. Vista dorsal. 9. Vista ventral. 10. Vista lateral. 11. Aedeagus. Barra, $1 \mathrm{~mm}$.
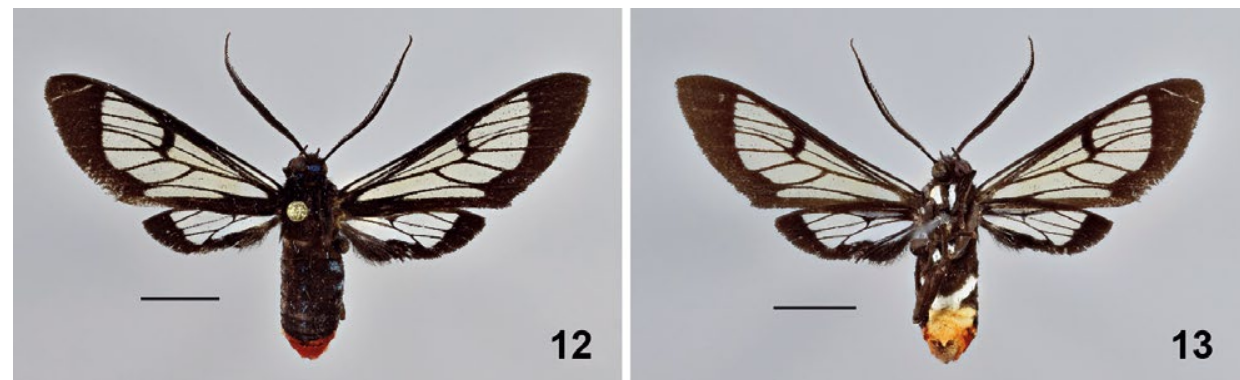

Figuras 12-13. Autochloris collocata. Macho (Brillo Nuevo). 12. Vista dorsal. 13. Vista ventral. Barra, $5 \mathrm{~mm}$.

12. Schaus (1924) describió a Autochloris solimoes con ejemplares recolectados en la boca del río Tefé y río Solimões (Amazonas), ambos en Brasil. Es de tamaño pequeño y presenta características muy diferentes al género Autochloris Hübner. Es común capturarla con las trampas de luz. Presenta una marcada variación intraespecífica. Algunos ejemplares presentan el tórax y el abdomen negros, salvo los dos puntos rojos en las partes laterales del primer segmento abdominal, en tanto otros, presentan las tégulas y puntos rojos en las partes laterales de todos los tergites abdominales. Es muy similar a Pheia nanata Kaye. Sin embargo, no contamos con evidencia suficiente para aseverar que ambas especies sean coespecíficas. 

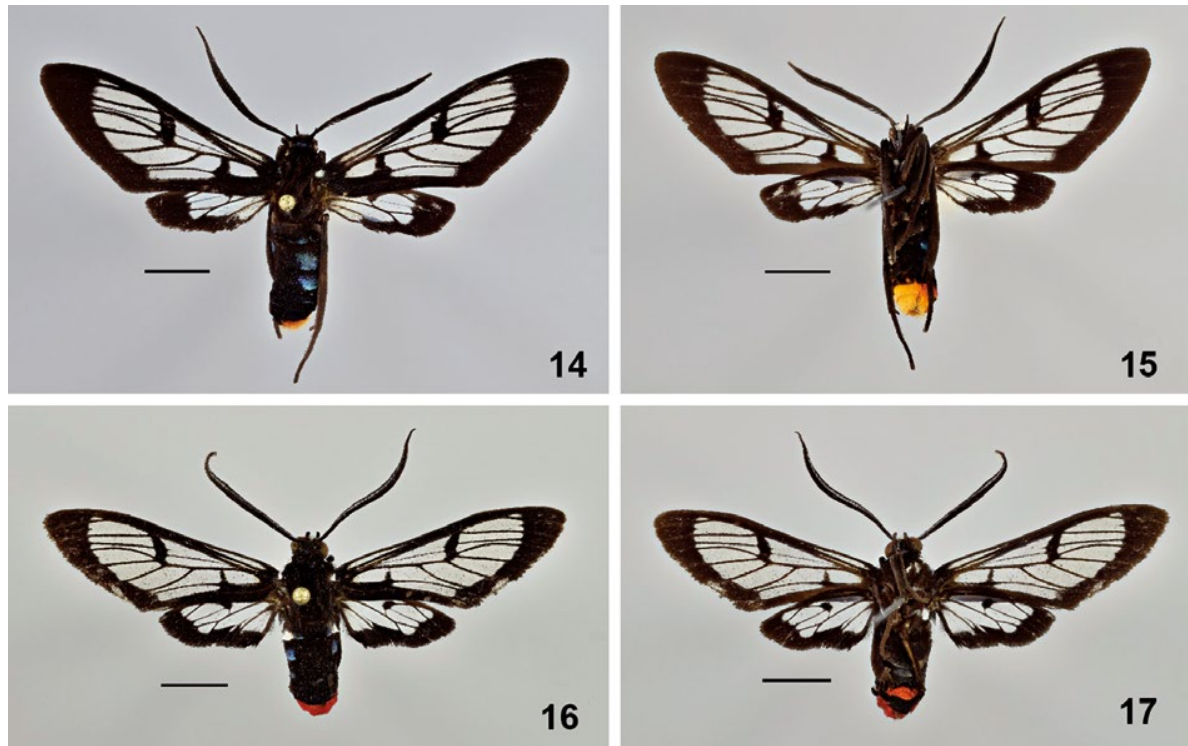

Figuras 14-17. Machos de Autochloris consociata y A. bijuncta. Fig. 14-15. A. consociata (San Juan de Poli). 14. Vista dorsal. 15. Vista ventral. Fig. 16-17. A. bijuncta (Saint Laurent, Guyana Francesa). 16. Vista dorsal 17. Vista ventral. Barra, $5 \mathrm{~mm}$.
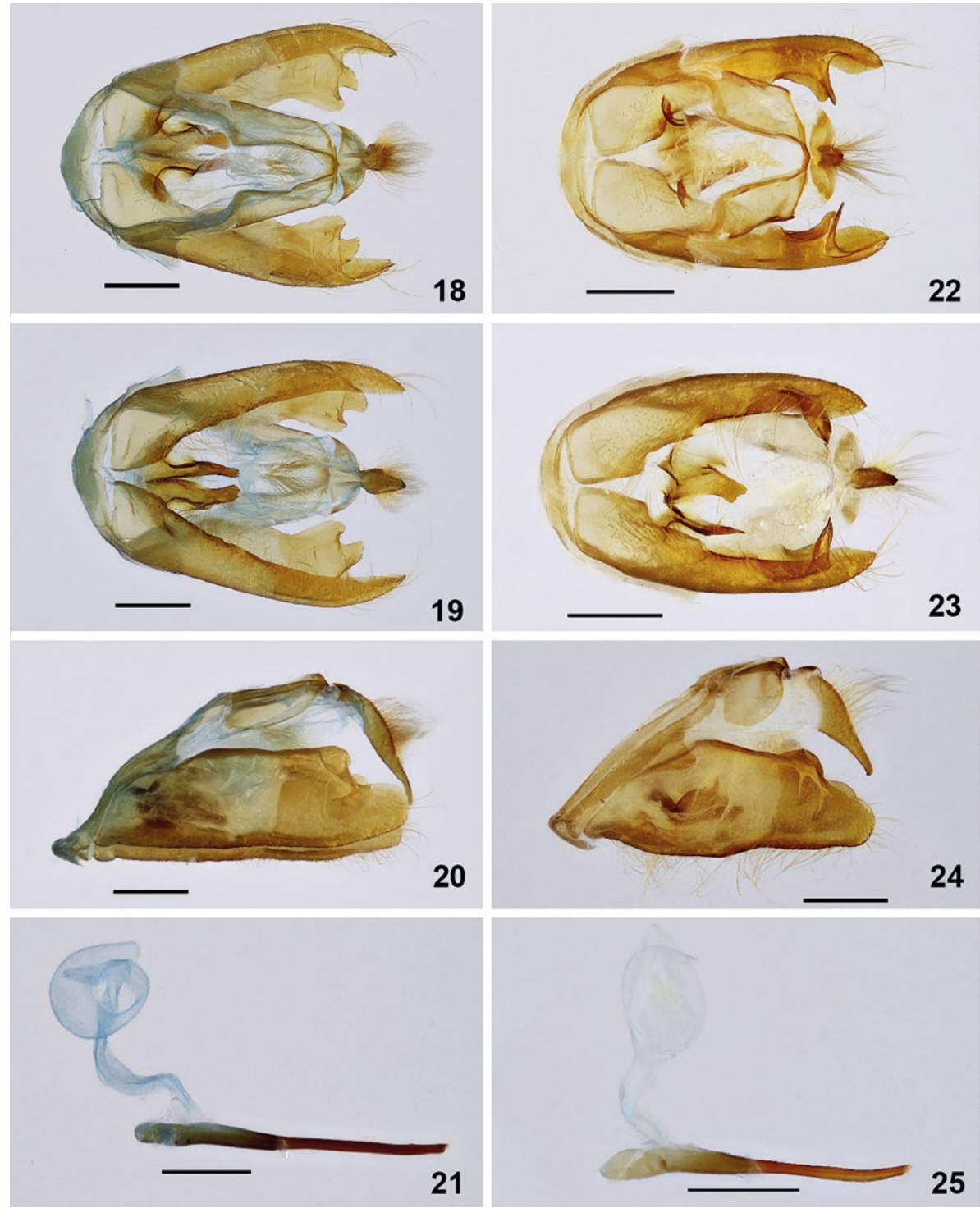

Figuras 18-25. Genitales machos de Autochloris consociata y A. bijuncta. Fig. 18-21. A. consociata (Genitalia \# JGA-1106 MUSM) (San Juan de Poli). 18. Vista dorsal. 19. Vista ventral. 20 Vista lateral. 21. Aedeagus. Fig. 22-25. A. bijuncta (Genitalia \# JGA-1113 MUSM) (Saint Laurent, Guyana Francesa). 22. Vista dorsal. 23. Vista ventral. 24. Vista lateral. 25. Aedeagus. Barra, $1 \mathrm{~mm}$. 
13. Sarosa ignicornis fue descrita con solo un ejemplar macho con localidad tipo Yahuarmayo. En una de las etiquetas del holotipo (NMHUK), solo se lee "Yahuarmayo / Perú / $1200 \mathrm{ft}$ / April - May 1912". La localidad con seguridad se refiere al río Yahuarmayo (tributario del río Inambari), localizado en los bosques montanos del departamento de Madre de Dios y no a la localidad Yahuarmayo en la sierra del departamento de Cusco. La especie es idéntica a Sarosa boenninghauseni descrita por Rothschild (1911), quien no estuvo seguro de la procedencia del ejemplar que consideró para la descripción, y que en su publicación solo anotó "Province of Rio de Janeiro ?". Esto último pone en duda si la especie en realidad ocurre en el sudeste de Brasil o es un error de etiquetado. Al menos, hasta ahora la especie no ha sido registrada para el área aludida (Teston \& Corseuil 2004, Ferro, V.G. \& I.R. Diniz 2007).

14. Tigrinadia quadricincta Kaye, 1918 (Figs. 26-34) = Isanthrene flavizonata Gaede, 1926. Nuevo sinónimo. Kaye (1918) propuso el género monotípico Tigrinadia, mencionando que es cercano a Sarosa Walker, diferenciándose por la venación de las alas anteriores. Describió la especie Tigrinadia quadricincta con un ejemplar hembra (colección Joicey) (MNHUK) proveniente del río Ucayali. La especie Isanthrene flavizonata fue descrita por Gaede (1926) con un ejemplar macho (ZMHU) proveniente de Iquitos. El ejemplar fue recolectado por Otto Michael, siendo probable que haya sido recolectado en los alrededores de la actual ciudad de Iquitos. Los ejemplares de ambas especies presentan los mismos caracteres morfológicos, patrón de coloración, siendo a su vez simpátricas. En nuestras recolectas, se tiene ejemplares machos y hembras de varias localidades en los alrededores de Iquitos y, la hembra coincide plenamente con la hembra descrita del río Ucayali. Proponemos que ambas especies son coespecíficas, siendo en este caso I. flavizonata un nuevo sinónimo de Tigrinadia quadricinta. Los registros más meridionales de esta especie consisten de un ejemplar macho de Contamana (Loreto, río Ucayali) y una hembra de Panguana (Huánuco, río Pachitea). Por ahora solo se conoce de Perú. En los Euchromiina, Hampson (1898) se refirió a los órganos androconiales ubicados en los primeros segmentos del abdomen como "valva ventral" y fueron descritos en detalle por Barth (1953). Existen dos tipos de órganos androconiales relacionados a las modificaciones de los primeros segmentos abdominales, para los cuales Weller et al. (2000) propusieron llamar como "bolsillo simple" para la modificación del segundo y tercer segmento, y "bolsillo doble" para la modificación del segundo, tercero y cuarto segmento. Tigrinadia quadricinta presenta un órgano androconial diferente a los conocidos hasta ahora. No son fácilmente visibles como los dos tipos mencionados. Solo son observables con tratamiento del abdomen con $\mathrm{KOH}$. El órgano andrococial es una modificación de la parte anterior y central del tercer tergite abdominal; es una invaginación donde se encuentra la floculencia característica de los bolsillos subabdominales (Fig. 34). Con el objetivo de evitar confusiones, proponemos para este tipo de órgano androconial como "bolsillo subabdominal tipo III", quedando los nombres de tipo I y II, para el bolsillo subabdominales simple y doble, respectivamente.
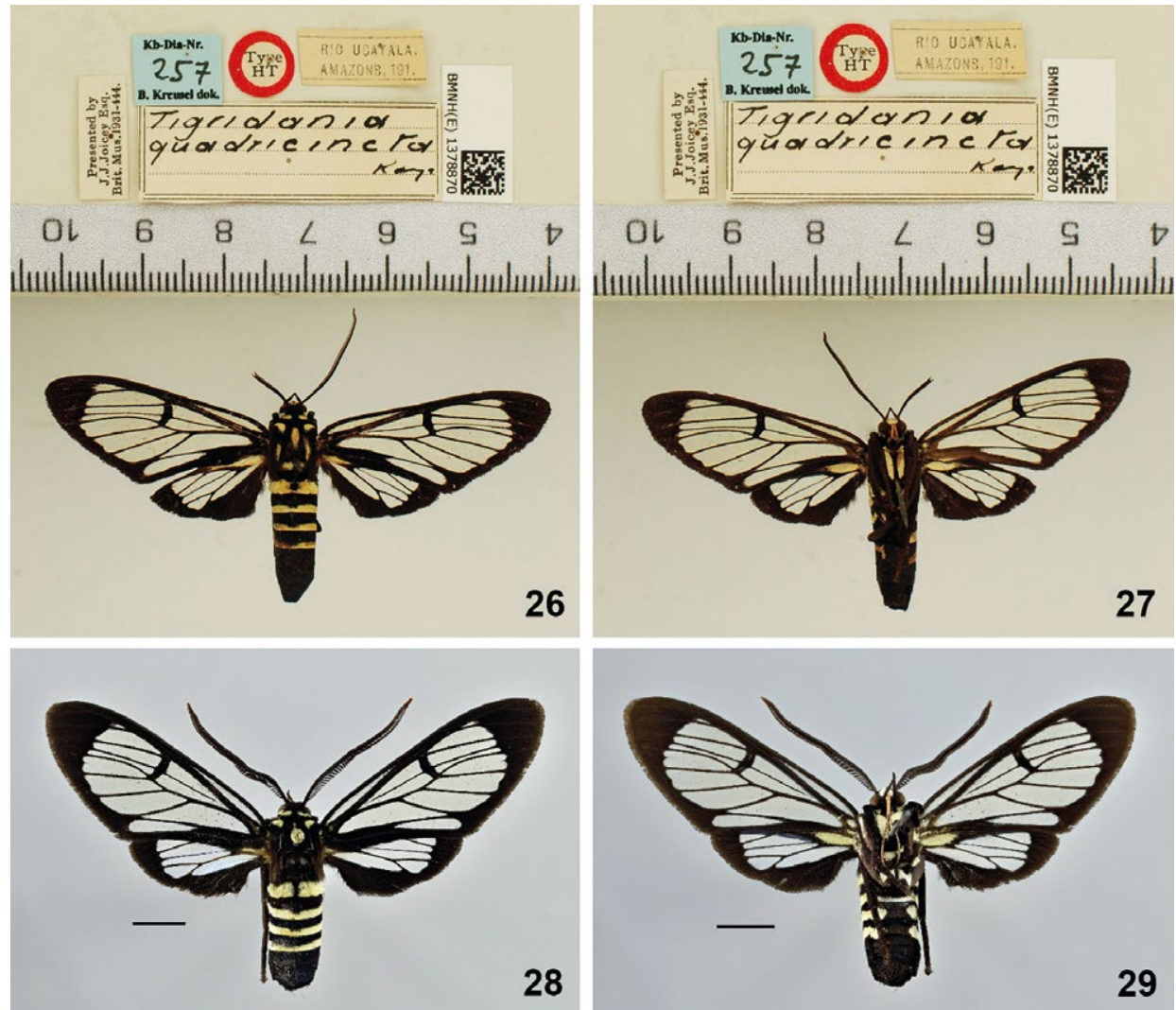

Figuras 26-29. Tigrinadia quadricincta. Fig. 26-27. Holotipo hembra (NMHUK). 26. Vista dorsal. 27 Vista ventral. Fig. 28-29. Macho (Centro Fuerte). 28. Vista dorsal. 29. Vista ventral. Barra, $1 \mathrm{~mm}$. 




Figuras 30-34. Genital y órgano androconial del macho de Tigrinadia quadricincta (Genitalia \# JGA-1104 MUSM) (Centro Fuerte). 30. Vista dorsal. 31. Vista ventral. 32. Vista lateral. 33. Aedeagus. 34. Órganos androconial en el tercer esternite del abdomen. SII, esternite II; SIII, esternite III; SIV, esternite IV. Barra, $1 \mathrm{~mm}$.
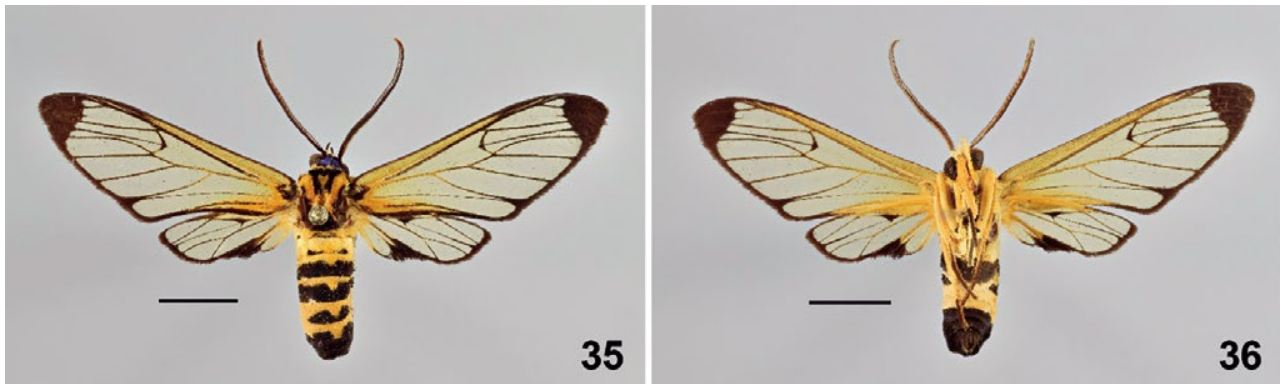

Figuras 35-36. Andrenimorpha eusebia. Macho (Qda. Rumiyacu). 35. Vista dorsal. 36. Vista ventral. Barra, $5 \mathrm{~mm}$.

15. Andrenimorpha eusebia (Figs. 35-36) fue descrita de Sarayacu (Ecuador). Es un nuevo registro para el Perú y que por ahora solo ocurre en Loreto.

16. Andrenimorpha lycopolis fue descrita de una ejemplar (probablemente hembra) de Sarayacu (Ecuador), al este de Ecuador. Es de hábito diurno, fue recolectado con trampas de Heliotropium sp. Sin embargo, algunos ejemplares también fueron atraídos a las trampas de luz. Es un nuevo registro para Perú.

17. Bryk (1953) decribió a Psoloptera leucotmemica basandose en 2 machos y 2 hembras del río Autaz (Brasil). De acuerdo con los caracteres morfológicos externos y la morfología de la genitalia, la especie pertenecería al género Leucotmemis. Los especímenes que ocurren en Loreto son muy parecidos a la descripción de Leucotmemis leucotmemica, salvo por algunos caracteres en el patrón de coloración. El holotipo está depositado en el Museo de Historia Natural de Suecia. Sería muy útil conocer la morfología de la genitalia, con el objetivo de poder discernir algunas poblaciones que ocurren en la Amazonía y que son muy semejantes externamente a la especie descrita por Bryk.

18. Leucotmemis torrida fue descrita de un ejemplar hembra de la colección Bates y recolectada en Tapajoz (Brasil). Es un nuevo registro para Perú.

19. Cosmosoma contracta fue descrita de un ejemplar macho (holotipo en UMO). La descripción original proporciona como localidad tipo solo como "Valley of the Amazons". Muy peculiar por presentar el cuerpo de color negruzco con tonalidad azulada iridiscente, alas transparentes con venas marrones, manchas anaranjadas, margen posterior y termen marrón que llegan al ápex. 
C. contracta (Figs. 37 - 42) es muy similar a C. gemmata Butler. Esta última conocida de Santa Marta (Colombia) y presenta la mancha marrón del termen y el ápex más anchas. Lo más probable es que haya sido recolecta en Brasil. La consideramos como un nuevo registro para Perú.

20. El género Mystrocneme Herrich-Schäffer fue propuesto por Herrich-Schäffer con la especie tipo M. geminata, la cual es considerada como un sinónimo más reciente de $M$. varipes Walker. Actualmente el género consta de 4 especies: $M$. varipes Walker, M. atavia Hampson, M. sectum Kaye y M. albicorpus Kaye (Dyar, 1907 Draudt, 1916). Druce (1889) describió a Saurita mecrida con un ejemplar hembra de la ciudad de México. Años más tarde, Mystrocneme dulcicordis fue descrita por Dyar (1907) con un macho, también recolectado en la ciudad de México. La especie $S$. mecrida no presenta dimorfismo sexual, salvo lo que se observa en casi todas las especies, el tamaño de los flagelómeros de las antenas. Hampson (1914) consideró la especie M. dulcicordis dentro del género Rhynhcopyga Felder, mientras que Draudt (1916) la consideró como un sinónimo más reciente de $S$. mecrida (actualmente como Pseudomya mecrida). A pesar de lo controvertido de la posición taxonómica de la especie, con seguridad no pertenece al género Mystrocneme. Nuestra hipótesis es que el género Mystrocneme es parafilético. Existen otras especies catalogadas dentro de otros géneros y que comparten caracteres con $M$. varipes, como es el caso de Chrostosoma orathidia (Druce, 1898) entre algunas otras especies.

21. Pseudomya biradiata (Figs. 43-48) fue descrita por Felder (1869) de un número de ejemplares no especificados y recolectados por H.W. Bates en la Amazonía de Brasil. Un ejemplar macho etiquetado como tipo se encuentra en el NHMUK. Por otro lado, $P$. hemiphaea fue descrita por Dognin (1909) de un ejemplar macho de Maroni (Guyana Francesa), esta especie ha sido confirmada por Cerda (2008) en la Guyana Francesa, quien dio a conocer la morfología del genital. Al revisar los holotipos, ambas especies son muy similares en cuanto a la morfología externa. Sin embargo, los genitales son algo diferentes con relación al aedeagus, por lo que consideramos por ahora, ambas especies como válidas. Es un nuevo registro para Perú.
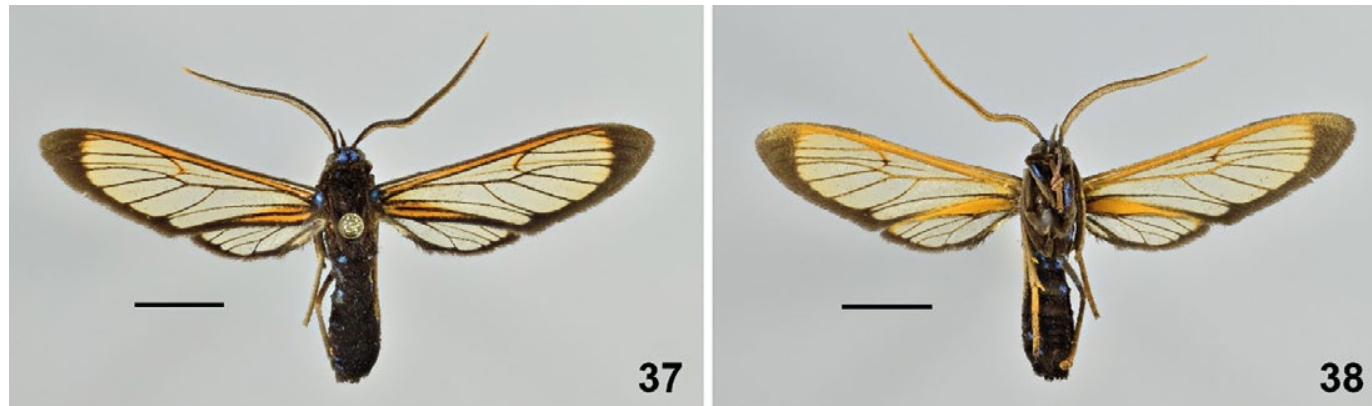

Figuras 37-38. Cosmosoma contracta. Macho (San Juan de Poli). 37. Vista dorsal. 38. Vista ventral. Barra, $5 \mathrm{~mm}$.
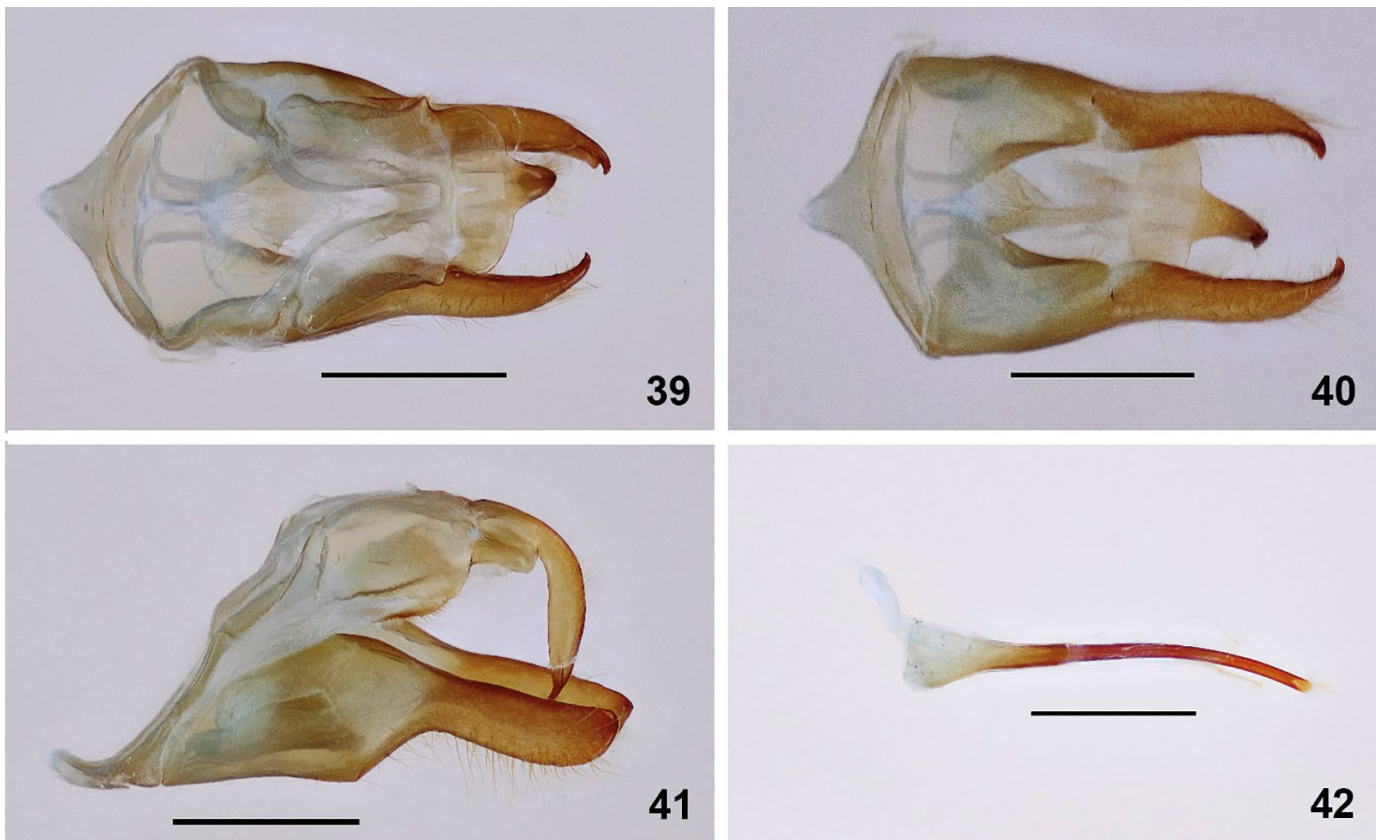

Figuras 39-42. Genital macho de $C$. contracta (Genitalia \# JGA-1109 MUSM) (San Juan de Poli). 39. Vista dorsal. 40. Vista ventral. 41. Vista lateral. 42. Aedeagus. Barra, $1 \mathrm{~mm}$. 


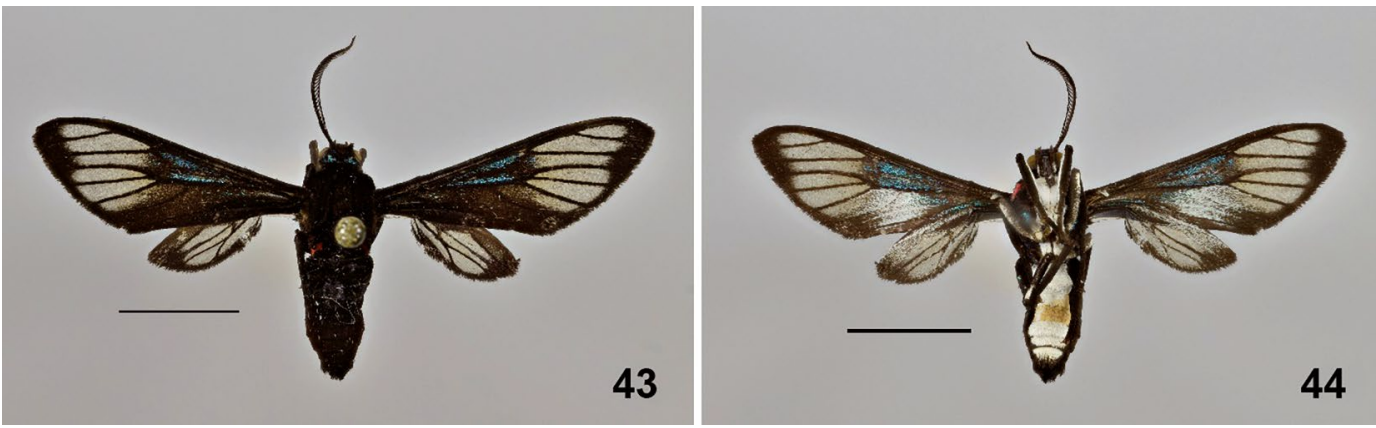

Figuras 43-44. Pseudomya biradiata. Macho (11.5 km NO de Huatape). 43. Vista dorsal. 44. Vista ventral. Barra, $5 \mathrm{~mm}$.

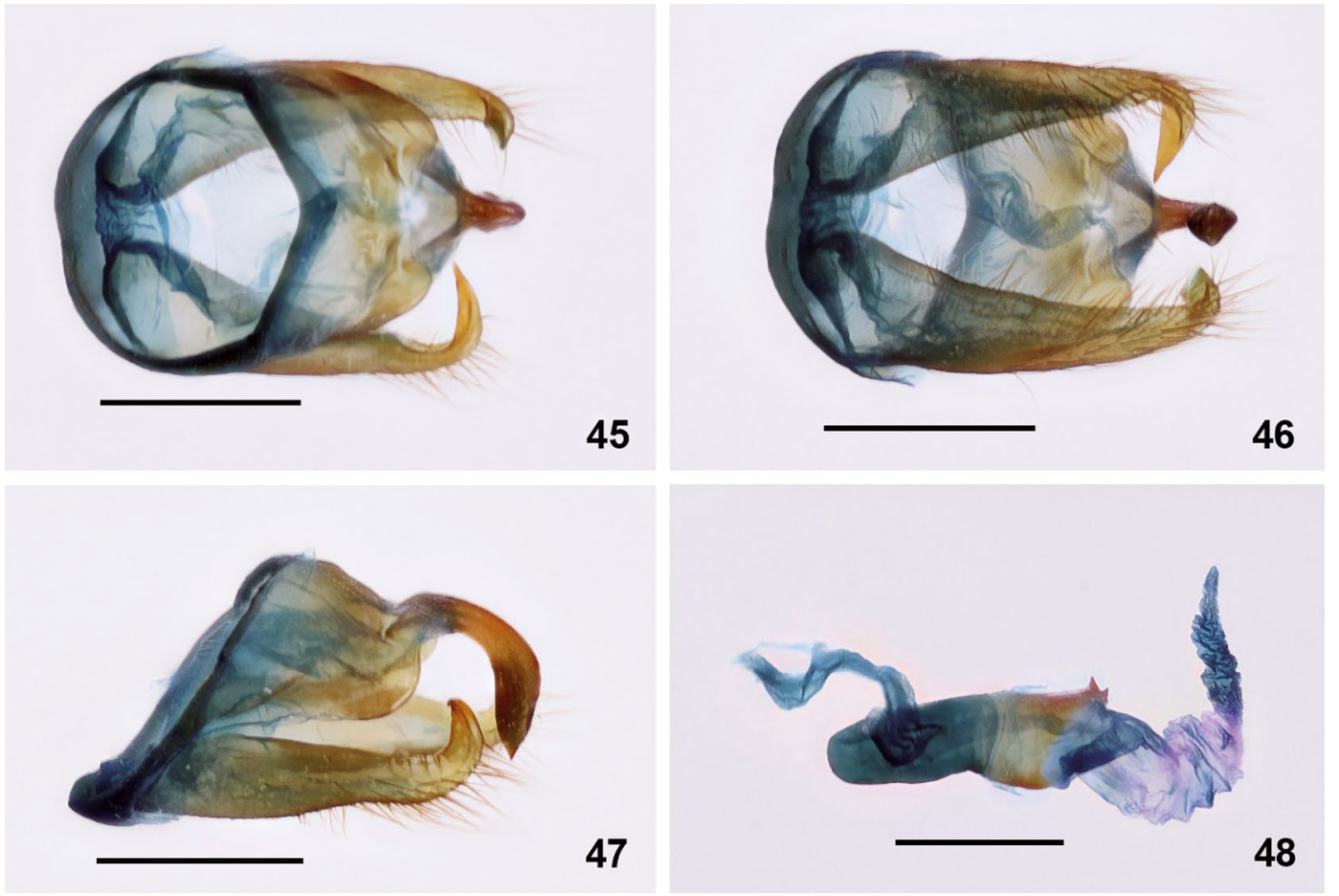

Figuras 45-48. Genital macho de Pseudomya biradiata (Genitalia \# JGA-1112 MUSM) (11.5 km NO de Huatape). 39. Vista dorsal. 40. Vista ventral. 41. Vista lateral. 42. Aedeagus. Barra, $1 \mathrm{~mm}$.

22. Pseumodya cretheis fue descrita de Sarayacu (Ecuador). Es un nuevo registro para Perú y conocida por ahora solo de Loreto.

23. Pseudomya hilda fue descrita de La Oroya (Puno, Perú) con material recolectado por G.R. Ockenden. Por ahora, los registros de Loreto son los más septentrionales encontrados.

24. Pseudomya vindonissa es común en toda la Amazonía peruana. Presenta dos morfotipos: el más abundante, con el tórax y los primeros segmentos abdominales rojo-anaranjado; la forma melánica, más rara, todo oscura, a excepción de la presencia de los puntos blancos en el tórax y abdomen. Esta última reportada como forma albipuncta por Gaede (1926), justamente de Pebas (Loreto) (Figs. 49-56).

\section{Discusión}

La paradoja científica peruana es que siendo considerado el Perú un país megadiverso, se tiene solo aceptable conocimiento de la diversidad biológica de las plantas y vertebrados (Ministerio del Ambiente 2019), lo que contrasta con nuestro escaso o nulo conocimiento acerca de la mayoría de los invertebrados. Haciendo referencia dentro de los insectos al Orden Lepidoptera, tenemos que de las más de 80 familias que ocurren en Perú, solo se está estudiando cerca de una docena de familias (Aguilar, 1995). Esta realidad es reflejada en nuestro conocimiento de los Lepidoptera en el departamento de Loreto. En el caso de las mariposas, solo se tiene el trabajo sobre Papilionoidea y Hesperoidea llevado a cabo en el alto río Napo, donde se registró 673 especies (Lamas et al., 1996) y una lista preliminar de los Saturniidae (Racheli \& Callegari 1996), ambos con una antigüedad de 25 años. 



Figuras 49-52. Machos de Pseudomya vindonissa. Fig. 49-50. Macho (Río Tapiche). 49. Vista dorsal. 50. Vista ventral. Fig. 51-52. Macho, morfotipo oscuro (Allpahuayo-Mishana). 51.Vista dorsal. 52. Vista ventral. Barra, $5 \mathrm{~mm}$.
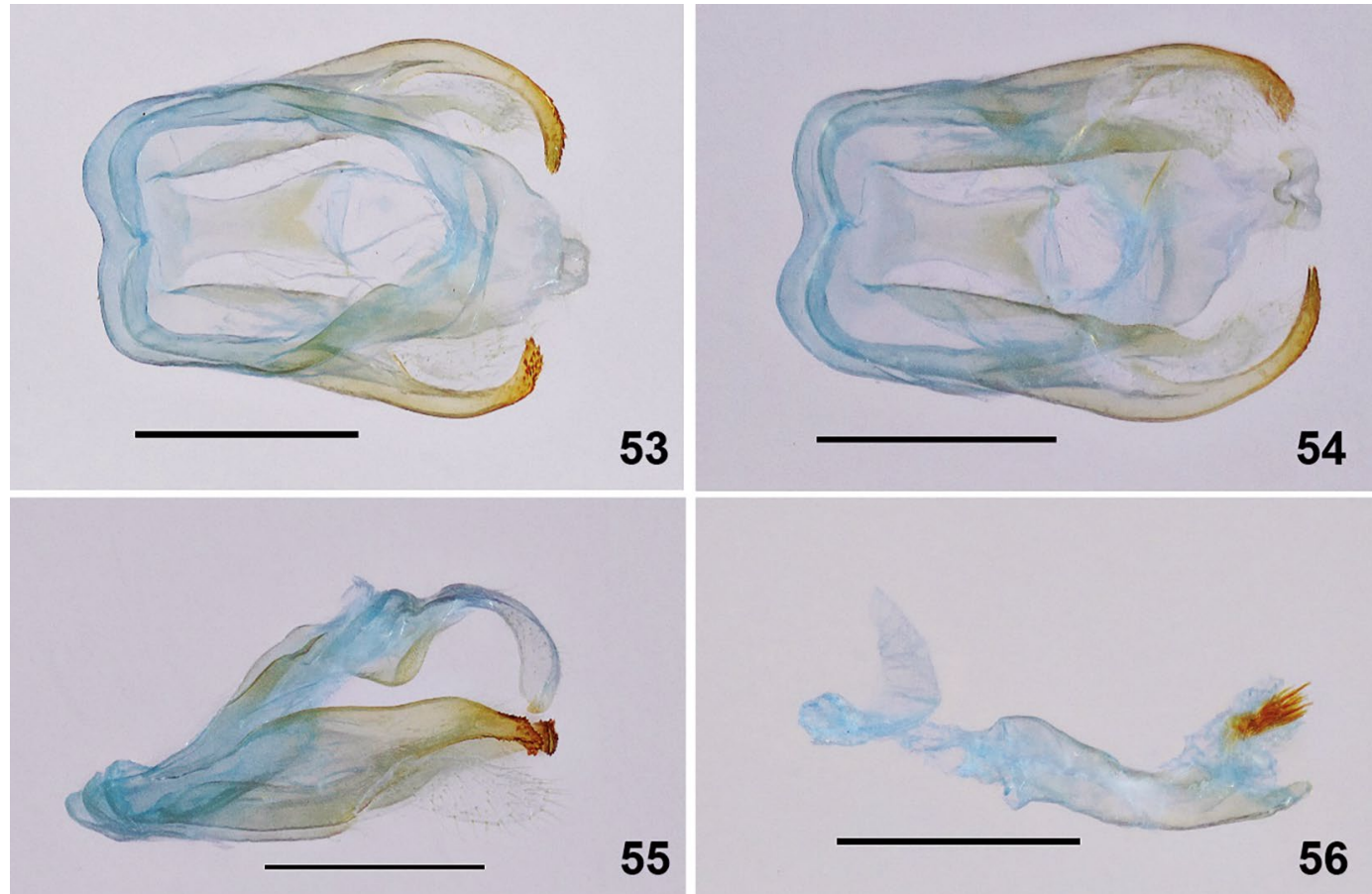

Figuras 53-56. Genital macho de P. vindonissa (Genitalia \# JGA-401 MUSM). 39. Vista dorsal. 40. Vista ventral. 41. Vista lateral. 42. Aedeagus. Barra, $1 \mathrm{~mm}$.

Investigaciones sobre Euchromiinae en Loreto no se han llevado a cabo; sin embargo, en el Perú se cuenta con las llevadas a cabo en Tambopata, donde se registró 72 especies (Grados 1999), para el bajo río Urubamba 91 especies (Grados 2001) y para dos lugares en el Parque Nacional Bahuaja-Sonene con 50 especies (Grados et al. 2015). Para otros lugares de la región Neotropical, se pueden mencionar para el Estado de Veracruz (México), donde se registró 66 especies (Hernádez-Baz \& Grados 2004); en Brasil, para la ecorregión El Cerrado se reportó la ocurrencia de 76 especies (Ferro et al. 2010); por último, se tiene el reporte de 219 especies, basados en la literatura y colecciones de Brasil, para toda la Amazonía Brasilera (Teston \& Gianluppi 2019).

Con seguridad la riqueza de especies de Loreto es mucho mayor que la registrada en este trabajo. Es necesario al menos trabajos faunísticos en lugares determinados con varias recolectas durante al menos un año. Alternar trampas de luz, con búsqueda directa y la utilización de plantas como cebos que contengan pyrrolizidina. Al menos se conoce que cerca del $20 \%$ de las especies de Arctiinae son atraídas por los cebos de Heliotropium y que no llegan a la trampa de luz (Grados et al. 2015). 
Específicamente en los Euchromiinae, en el mismo trabajo, se encontró que el $16 \%$ de las especies no llegan a la trampa de luz y sí a las plantas de Heliotropium, especies como Dixophlebia quadristrigata (Walker), Rhynchopyga garleppi (Gaede) y Sphecosoma surrentum Druce. Casualmente esta última con el mismo patrón de comportamiento registrado en este trabajo.

Con la disponibilidad de especímenes de diferentes lugares ha sido posible la determinación de dos nuevos sinónimos. Estamos seguros de que existen muchos más en la región Neotropical. Estos se irán descubriendo con la utilización de herramientas moleculares (Laguerre 2016).

Los nueve nuevos registros para Perú, son importantes para entender los patrones de distribución de las especies y la verdadera riqueza que alberga nuestro país. Varias especies que han sido nuevos registros para Perú, en el país solo se han registrado en Loreto: $S$. abnormis, $A$. consociata, A. collocata, A. eusebia, P. critheis y P. biradiata. Por último, Tigrinadia quadricincta por ahora es una especie conocida solo de Perú.

Los bosques Amazónicos de Loreto albergan una alta riqueza de especies de Euchromiina que aún están por registrar, especies nuevas por describir (Grados datos inéditos), nos falta conocer el dimorfismo sexual de las especies, sus hábitos de comportamiento, la morfología de los estadios larvales, sus hospederos, la estacionalidad de las especies, sus diferentes adaptaciones morfológicas, y cómo afectó desde el Mioceno, hace 23 millones de años, la existencia del gran lago Pebas (Wasselingh \& Salo 2006, Hoorn et al. 2010) en los procesos evolutivos y los patrones de distribución geográfica que recién empezamos a conocer. Todo este conocimiento de la biodiversidad de un grupo tan interesante e importante de los insectos ayudará en la toma de decisiones para lograr en el mediano y largo plazo un manejo sustentable de la Amazonía.

\section{Literatura citada}

Aguilar, PG, Raven KG, Lamas G, \& Redolfi I. 1995. Sinopsis de los hexápodos conocidos del Perú. Revista peruana de entomología, 37: 1-9.

Cerda J. 2008. Euchromiini de Guyane Française. Lepidoptera: Arctiidae, Arctiinae. 171 pp. + I-III + 3 pls. [Publicado por el autor].

Comstock JH, Needman JG. 1898. The wings of insects. American Naturalist 32(373): 43-48; 32(374): 81-89; 32(376): 231-257; 32(377): 335-340; 32(378): 413424; 32(380): 561-565; 32(382): 769-777; 32(384): 903-911.

Comstock JH, Needman JG. 1899. The wings of insects. American Naturalist, 33(386):117-126; 33(391): 573-582; 33(395): 843-860.

Dietz RE, Duckworth D. 1976. A Review of the Genus Horama Hübner and Reestablishment of the Genus Poliopastea Hampson (Lepidoptera: Ctenuchidae). Smithsonian Contributions to Zoology, 215: $53 \mathrm{pp}$.

Dietz RE. 1994. Systematics and Biology of the genus Macrocneme Hübner (Lepidoptera: Ctenuchidae). University California Press, Entomology 113: 121 pp + 236 figs.
Draudt M. 1916-1919. Familie: Syntomidae. In: A. Seitz (Ed.), Die Gross-Schmetterlinge der Erde. Stuttgart, A. Kernen. 6: 33-230.

Ferro VG, Diniz IR. 2007. Arctiidae (Insecta: Lepidoptera) da Estação Biológica de Boracéia (Salesópolis, São Paulo, Brasil). Biota Neotropica, 7(3): 331-338.

Ferro VG, Melo AS, Diniz IR. 2010. Richness of tiger moths (Lepidoptera: Arctiidae) in the Brazilian Cerrado: how much do we know? Zoologia, 27(5): 725-731.

Fleming H. 1959. The Ctenuchidae (Moths) of Trinidad, B.W.I. Part. II. Ctenuchidae. Zoologica, 44 (3): 85-104, 3 plates.

Forbes WTM. 1939. The Lepidoptera of Barro Colorado Island, Panama. Bulletin of the Museum of Comparative Zoology, 84 (4): vii+97-322, 8 plates.

Gaede M. 1926. Amatiden des Berliner Zoologischen Museums. (Lep.). Deutsche Entomologische zeitschrift, 2: 113136.

Grados J, Baynes H, Razuri E, Figueroa L, Barrientos J, Guillermo E. 2015. Insectos. Invisiblemente mágico, pp. 92-107. En: Parque Nacional Bahuaja Sonene. Inventarios Biológicos Rápidos. M. Montoya, D. Cossios, M. Silva \& D. Coll (Editores), Wildlife Conservation Society, 119 pp.

Grados J. 2015. Histioea magistrae sp. nov. (Lepidoptera: Erebidae: Arctiini) de la Amazonía sudeste de Perú. Revista peruana de Biología 22(3):297-302.

Hampson G. 1914. Catalogue of the Lepidoptera Phalaenae in the British Museum. Supplement, vol. 1. British Museum (Natural History), London: xxviii + 858 pp.

Hampson GF. 1898. Catalogue of the Syntomidae in the collection of the British Museum. London, British Museum (Natural History). 1: xxii + 559 pp, 17 pls.

Hoorn C, Wesselingh FP, Hovikoski J, Guerrero J. 2010. The development of the Amazonian mega-wetland (Miocene; Brasil, Coloombia, Peru, Bolivia), pp. 123-142. In: Amazonia: Landscape and species evolution: A look into the past. C. Hoorn \& F.P. Wesselingh (editors), Blackwell Publishing Ltd.

Jacobson NL, Weller SJ. 2002. A cladictic study of the Arctiidae (Lepidoptera) by using characters of immatures and adults. Thomas Say publications in Entomology, Monographs. Entomological Society of America, $98 \mathrm{pp}$.

Kaye WJ. 1913. A few observations in mimicry. Transaction of the Entomological Society of London, pp. 1-10.

Kirby W. 1837. The insects. Parte 4. In: Fauna Boreali-Americana; or the zoology of the northern parts of British America: containing descriptions of the objects of natural history collected on the late northern land expeditions, under command of captain sir John Franklin, R.N. J. Richardson [ed.]. Josiah Fletcher, Norwich, London.

Kitching IJ, Rawlins JE. 1998. The Noctuoidea, pp. 355-401. In. P. Kristensen (Ed.), Lepidoptera, moths and Butterflies, Vol. 1: Evolution, Systematics, and Biogeography. Walter de Gruyer, Berlin, Germany.

Klages EA. On the Syntomid Moths of Southern Venezuela collected in 1898-1900. Proceedings of the United States National Museum, 29: 531-552.

Klots AB. 1970. Lepidoptera. In: Tuxen, S.L. (Ed.), Taxonomist's Glossary of Genitalia in Insects. Munksgaard, Copenhagen, pp. 97-111.

Lafontaine JD, Fibiger M. 2006. Revised higher classification of the Noctuoides (Lepidoptera). Canadian Entomology, 
138: 610-635.

Lafontaine JD, Schmidt BC. 2010. Annotated check list of the Nouctoidea (Insecta, Lepidoptera) of North America north of Mexico. Zookeys 40(1): 239 pp.

Laguerre M. 2016. Sex pairing of three highly dimorphic Arctiinae (Lepidoptera: Erebidae) females in French Guyana and description of three new species from Brazil. Journal of Insect Biodiversity, 4(21): 1-26.

Lamas G, Robbins RK, Harvey D. 1996. Mariposas del alto Río Napo, Loreto, Perú (Lepidoptera: Papilionoidea y Hesperoidea). Revista peruana de Entomología, 39: 63-74.

Ministerio del Ambiente. 2019. Sexto Informe Nacional sobre Diversidad Biológica. La Biodiversidad en cifras. Industria Gráfica Cimagraf S.A.C., 51 pp.

Pinheiro LR, Duarte M. 2010. Revision of te Neotropical moth genera Mallodeta Butler and Erruca Walker, revalidated (Noctuidae, Arctiinae, Arctiini, Euchromiina). Zootaxa, 2573:1-34.

Pitman N, Vriesendorp C, Moskovits DK, von May R, Alvira D, Wachter T, Stotz DF, del Campo A, eds. 2011. Perú: Yaguas-Cotuhé. Rapid Biological and Social Inventories Report 23. The Field Museum, Chicago.

Racheli L, Callegari MC. 1996. Lista preliminare des Saturnidi del Dipartimento di Loreto, Peru. Entomofauna, 17(35): 477-492.

Rougerie R, Laguerre M. 2010. Un cas remarquable de dimorphisme sexuel révélé par les codes barres ADN chez une arctiide néotropicale (Lepidoptera: Arctiidae) Annales de la Société entomologique de France, 46(34):477-480

Simmons RB. 2004. Description of Sphecosma pattiannae, new species, with comments on its novel male androconial (Lepidoptera: Arctiidae: Arctiinae: Euchromiini). Zootaxa 519: 1-12.
Simmons RB. 2006. A revision of Psoloptera Butler, including a redescription of its known species (Arctiidae: Arctiinae: Euchromiini). Journal of the Lepidopterists' Society, 60(38): 149-155.

Teston J, Corseuil E. 2004. Diversidade de Arctiinae (Lepidoptera, Arctiidae) capturados com armadilha luminosa, em seis comunidades no Rio Grande do Sul, Brasil. Revista Brasileira de Entomología, 48(1): 77-99.

Teston JA, Ferro VG. 2019. Arctiini Leach, [1815] (Lepidoptera, Erebidae, Arctiinae) of the Brazilian Amazon. IV Subribe Euchromiina Butler, 1876. Biota Neotropica, 19(4): 1-16.

Vriesendorp C, Álvarez JA, Barbagelata N, Alverson WS, Moskovits DK, eds. 2007. Perú: Nanay-Mazán-Arabela. Rapid Biological Inventories Report 18. The Field Museum, Chicago.

Vriesendorp C, Schulenberg TS, Alverson WS, Moskovits DK, Rojas JI, eds. 2006. Perú: Sierra del Divisor. Rapid Biological Inventories Report 17. The Field Museum, Chicago.

Weller SJ, Simmons RB, Boada R, Conner WE. 2000. Abdominal modification ocurring in wasp mimics of the Ctenuchinae-Euchromiine clade (Lepidoptera: Arctiidae). Ann. ent. Soc. Am. 93(4): 920-928.

Wesselingh FP, Salo JA. 2006. A Miocene perspective on the evolution of the Amazonian biota. Scripta Geologica, 133: 439-458.

Zahiri R, Holloway JD, Kitching IJ, Lafontaine JD, Mutaten M, Wahlberg N. 2012. Molecular phylogenetics of Erebidae (Lepidoptera, Noctuoidea). Systematics Entomology, 37: 102-124.

Zahiri R, Kitching IJ, Lafontaine JD, Mutanen M, Kaila L, Holloway JD, Wahlberg N. 2010. A new molecular phylogeny offers hope for a stable family level classification of the Noctuidea (Lepidoptera). Zoologica Scripta, 40(2): 158-173.

Zerny H. 1912. Syntomidae. Lepidopterorum Catalogus 7:179 $\mathrm{pp}$
Agradecimientos / Acknowledgments:

A los curadores de los diversos museos que permitieron la revisión del material bajo su custodia. Jérôme Barbut (MHNP), Alberto Zilli (NHMUK), James Hogan (UMO) y Wolfram Mey (ZMHB). Karla Mantilla elaboró el mapa de los puntos de recolecta. Dos revisores anónimos contribuyeron en la mejora del manuscrito.

Conflicto de intereses / Competing interests:

Los autores no incurren en conflictos de intereses.

Rol de los autores / Authors Roles:

JG: conceptualización, investigación, escritura- preparación del borrador original, redacción-revisión y edición. JJR: investigación, escritura- preparación del borrador original, redacción-revisión y edición.

\section{Fuentes de financiamiento / Funding:}

Los autores declaran que este trabajo no recibió financiación específica.

\section{Aspectos éticos / legales; Ethics / legals:}

Los autores declaran no haber incurrido en aspectos antiéticos y que no omitieron normas legales en la investigación y preparación de este trabajo. 

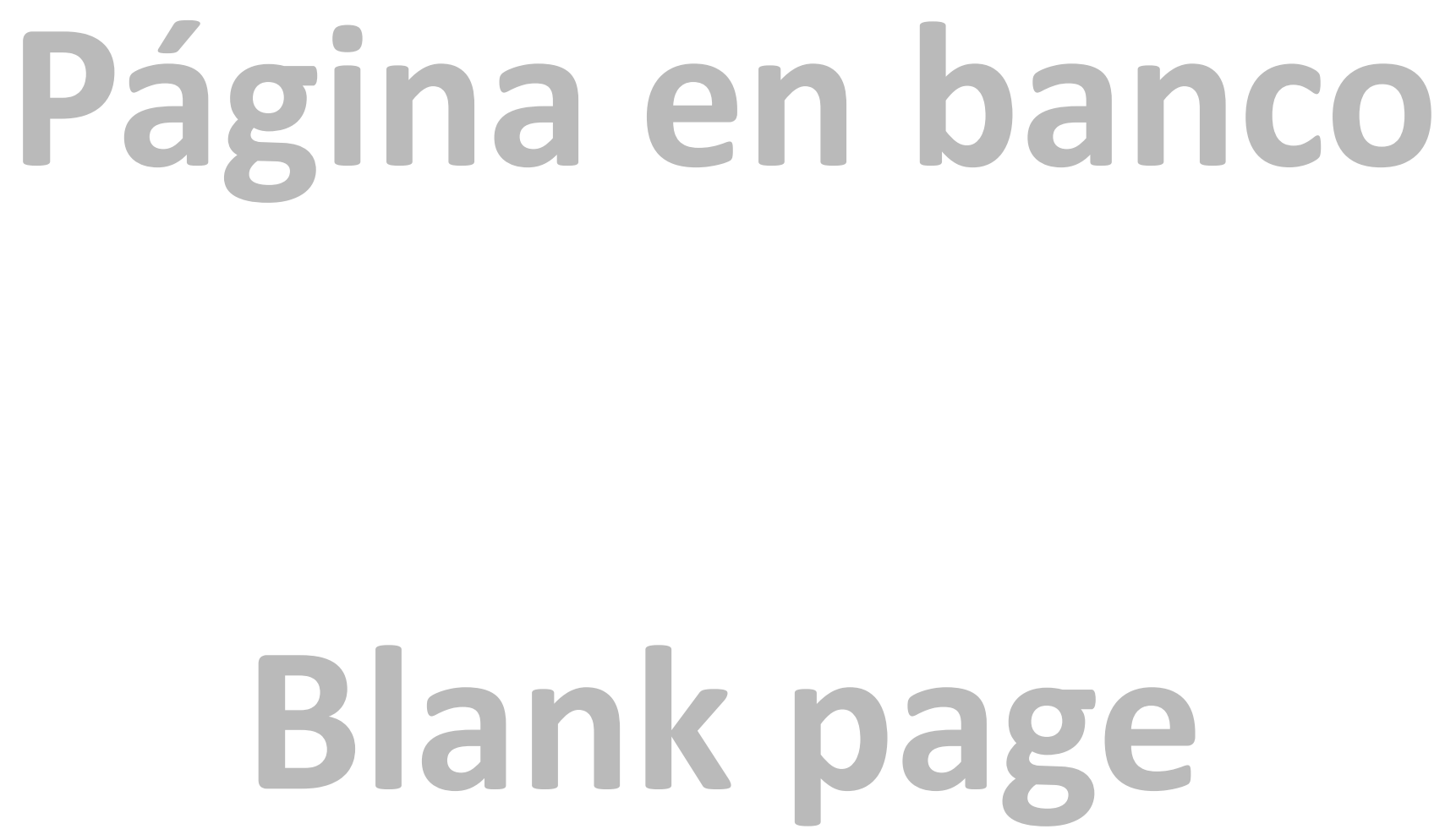